A Profit-Center Game With Incomplete Information

\author{
Tatsuro Ichiishi \\ Ohio State University \\ Roy Radner \\ Leonard N. Stern School of Business \\ New York University
}

August 1999

Working Paper Series

Stern \#IS-99-5 


\title{
A PROFIT-CENTER GAME WITH INCOMPLETE INFORMATION
}

\author{
Tatsuro Ichiishi ${ }^{1}$ \\ Department of Economics \\ Ohio State University \\ 1945 North High Street \\ Columbus, OH 43210-1172
}

\author{
Roy Radner ${ }^{a}$ \\ Stern School of Business \\ and \\ New York University \\ 44 West Fourth Street \\ New York, NY 10012
}

\begin{abstract}
Profit centers in a firm in multidivisional form agree in the ex ante stage upon a plan about their joint production and profit imputation. The plan is executed in the subsequent two periods of the interim stage. In the first interim period, each center has its private information, but a part of its information is revealed to the other centers through its action. Based on the information endogenously pooled this way, the centers take another round of actions in the second interim period. A core plan is defined as a Bayesian incentive-compatible plan of the grand coalition of profit centers, upon which no coalition can improve using its Bayesian incentive-compatible plan. A core plan is called full-information revealing if each center fully reveals its private information in the first period. Three existence theorems for a full-information revealing core plan are established. The first two theorems impose alternative conditions on returns to scale: (1) the neoclassical convex technology, and (2) increasing returns to scale. In case (2), a stronger condition than Scarf's distributiveness is imposed on the total production set. The third theorem is based on a specific supplier-customer relationship among the divisions.
\end{abstract}

JEL classification: D2, D8, L2

Keywords: Firm in multidivisional form; Transfer payment problem; Incomplete information; Core plan; Full-information revealing core plan; Asset specificity.

\footnotetext{
${ }^{1}$ Tel.: +1-614-292-0762; fax: +1-614-292-3906; email: ichiishi@ecolan.sbs.ohiostate.edu

${ }^{\circ}$ Corresponding author. Tel.: +1-212-998-0813; fax: +1-212-995-4228; email: rradner@stern.nyu.edu
} 


\section{Introduction}

A firm in multidivisional form (an M-form firm, in short) is a corporation in which several divisions (or profit centers) are operated semi-autonomously. The significance of $\mathrm{M}$-form firms in the present-day economy has increasingly been recognized since the publication of Chandler's (1962) seminal study of their historical development.

Each division in an $\mathrm{M}$-form firm is, to a significant extent, an independent decision-maker. ${ }^{2}$ As decision-units of the same corporation, however, these divisions talk to each other and coordinate their production activities. Total profit will then be distributed to the divisions. While coordination of production activities better serves interests of the divisions, there arise conflicts when it comes to the imputation of profit. The divisions therefore agree on a specific plan for coordinated activities/imputation, so that no coalition of divisions can improve upon it by its own effort, that is, the divisions are settled at a self-enforcing agreement. This is precisely the scenario that the descriptive strategic cooperative game theory analyzes. Recently, Radner (1992) (1) formulated the internal organization of an M-form firm as a static model of a profit-center game, (2) viewed the core of the game (a central descriptive cooperative solution concept) as the self-enforcing agreements, and (3) studied its properties for several interesting cases.

The history of strategic cooperative game theory, in particular the history of cooperative extensions of noncooperative games, is quite long by now. Here, it is only mentioned that, following the earlier work on the cooperative extensions of the Bayesian game, Ichiishi, Idzik and Zhao (1994) (1) addressed the issue of whether or not player $j$ in coalition $S$, in pursuit of his self-interest, decides to pass on his private information to the other members of $S$, and if he does, which part of his private information he decides to pass on, (2) proposed a new version of the Bayesian incentive compatible strong equilibrium concept which answers this issue endogenously, and (3) established generic existence theorems for this equilibrium.

The present paper addresses information processing in an M-form firm. Until recent years, the economics of information has focused on noncooperative behavior, in particular the Stackelberg-type relationship between a

\footnotetext{
${ }^{2}$ Of course, some decisions are typically made by the firm's central management, e.g., levels and types of capital expenditures, location, and even total numbers of employees.
} 
single mechanism-designer and several agents. This setup is inappropriate for analysis of information processing in an M-form firm, because there is no such relationship among the divisions. Indeed, all divisions participate in the design of a corporate mechanism, and then execute their tasks in accordance with the agreed upon mechanism.

Roughly stated, the present paper is built upon the works of Radner (1992) and Ichiishi, Idzik and Zhao (1994). The information studied here is about types of the divisions; the precise content of a type will be beriefly described in the third paragraph below, and will be spelled out in detail in paragraphs four through seven of Section 2. In the ex ante stage, divisions in an $\mathrm{M}$-form firm agree on a mechanism (called henceforth a plan), which specifies production of commodities and profit imputation contingent upon each type-profile. The plan is executed in the subsequent two periods of the interim stage. In the first interim period, each division has its private information on its true type, but by making specific supply/demand decisions about some commodities, it reveals to the other divisions that one of its types at which this specific supply/demand should be made according to the plan must be true. A part of the private information is endogenously pooled this way. Based upon the pooled information (in addition to the other part of information that still remains private), the divisions make supply/demand decisions about the other commodities and claim their profit imputation in the second interim period. To enforce divisions' truthful actions in each period, only Bayesian incentive-compatible plans are considered during the planning period of the ex ante stage. The headquarters of the firm may be considered one of the divisions that actively participate in coalitional design of a plan and subsequent execution of the agreed upon plan. It is postulated that the headquarters also plays the role of a risk-neutral insurer; even with this postulate, the divisions' behavior and hence the descriptive solution of the game reflect the intricate problem of information processing - for a more detailed discussion, see the paragraph that follows statement of Postulate 2.4. A core plan is defined as a Bayesian incentive-compatible plan of the grand coalition of divisions, upon which no coalition can improve using its Bayesian incentive-compatible plan. It is the Bayesian incentive-compatible strong equilibrium specialized to the present context. Due to the richness of the structure of the profit-center game, exact existence theorems can be established here, rather than merely generic existence theorems.

Actually, a stronger notion of core is considered here. A core plan is called 
full-information revealing if each division fully reveals its private information in the first period. Conditions for the existence of a full-information revealing core plan are established in the present paper.

One of the key ingredients in Radner' (1992) formulation of an M-form firm, which is also replicated in the present paper, is the distinction of market commodities and nonmarket commodities; while a commodity in the former category has a price established in the market outside the firm, a commodity in the latter category has no price and is used only internally. An intermediate nonmarket commodity is a commodity, not available in the market, which is supplied as an output by a division of the firm and is demanded as an input by another division. A central resource allocation problem in an M-form firm then arises: A nonmarket commodity produced or initially held by a division (say, division $i$ ) is transferred to another division (say, division $j$ ), and the two divisions have to come up with a mutually agreeable level of payment that $j$ has to make to $i$ in return for the use of the commodity. The core profit imputation concept addresses this problem.

Just as a division can produce an intermediate nonmarket commodity while some others cannot, production sets can differ among the divisions. This typically presupposes the asset specificity for each division, as emphasized with empirical evidence by Williamson (1975) and Klein, Crawford and Alchian (1978): Some assets are so specialized that the benefit from their use in the firm overweighs their salvage value in the market. Klein, et al. (1978) call the difference between the benefit and the salvage value the quasi rent value. When the quasi rent value is high, the firm does not bother to sell the assets as a scrap, so these resources are classified as nonmarket commodities. Williamson (1991) lists six kinds of asset specificity, but for simplicity of argument the present paper will focus on physical-asset specificity such as specialized plants designed for production of specific outputs and specialized dies required to produce an intermediate commodity, and the human-asset specificity that arises in learning by doing of the human-resource holders. Differences in production sets reflects differences in specialization. The degree of specialization may well be private information of division $i$, and this fact actually motivates the present Bayesian formulation of the M-form firm in the following two ways: First, a feasible net output depends on a typeprofile. Second, by interpreting the quantity of a nonmarket commodity $a$ 
[the actual physical quantity] $\times$ [its efficiency (degree of specialization)],

the amount of $a$ that division $i$ initially holds depends on $i$ 's type.

There are three existence results for a full-information revealing core plan in the present paper. For all results, nonmarket commodities that are supplied/demanded in the first interim period play a crucial role. The first result is under the neoclassical convexity assumption on the production sets (Theorem 3.1). It is consistent with the presence of intermediate nonmarket commodities, so the transfer payment problem is fully answered. The second result is for a particular class of total production sets which satisfy increasing returns to scale (Theorem 4.2). Here, a stronger condition than Scarf's (1986) distributiveness is imposed on the total production set $Y$. Moreover, the market commodities are assumed to be used only as inputs (so intermediate nonmarket commodities are excluded from the analysis). Roughly speaking, the set $Y$ is required to satisfy: (1) increasing returns to scale with respect to any equiproportional increase in all the type-profile-contingent commodities; (2) strict convexity of the input-requirement sets (in the space of nonmarket input plans); and (3) strict convexity of the auxiliary concept of production possibility sets (in the space of market net output plans) defined in terms of the hypothetical, derivative concept of "cost function." A noncommodity resource of a division is a resource owned by or assigned to that division, which is not for sale (so it is not a market commodity), and which can not be transferred to another division (so it is not a nonmarket commodity); e.g., a plant of each division. It should be pointed out that conditions (1) and (3) together implicitly assume that each net output plan needs the associated adjustment of non-commodity resources and/or effort. In spite of the inability to analyze the role of intermediate nonmarket commodities in the second result (Theorem 4.2), the transfer payment problem for exchange of initial nonmarket resources is explicitly solved by a core profit imputation plan. The third result is for a particular structural relationship among the divisions (Theorem 5.2). Here, the divisions are divided into the suppliers and the customers. The suppliers produce and supply to the customers nonmarket intermediate commodities. The customers use these nonmarket intermediate commodities, produce market commodities and bring in profit to the firm. No assumption is made in Theorem 5.2 on returns to scale. 
A core plan is a descriptive solution concept; it describes the mechanism that the divisions determine endogenously. Its non-optimality property was studied in Ichiishi and Sertel (forthcoming, Example 4.4). Section 2 of the present paper reports very briefly the main conclusions of Ichiishi and Sertel (forthcoming, Example 4.4) on the non-optimality.

The next section constructs the model of profit-center game with incomplete information, and presents basic postulates and assumptions. While it contains a precise formal treatment of the model and key assumptions, every attempt is made to present verbally the economic motivations for the concepts and the economic meanings or justifications of the assumptions. Some concepts are straightforward to motivate, yet require much involved technical formulations for precision; in such cases a formal definition will be postponed until Section 6. In Section 3, an existence theorem is stated for the neoclassical case of convex technology. In Section 4, an existence theorem is stated for the case in which increasing returns to scale prevail. In Section 5 , an existence theorem is stated for the case in which a specific supplier-customer relationship holds among the divisions. Section 6 contains complete proofs of the theorems.

\section{A Multidivisional Firm with Incomplete Information}

The transfer payment problem that arises in Chandler's (1962) firm in multidivisional form was formulated by Radner (1992). This section extends Radner's model to the situation in which each division may have its private information. The divisions determine in the ex ante stage a mechanism that specifies their actions and rewards in the subsequent interim stages contingent upon the reported (that is, pretended) or inferred types. In the present context of an M-form firm, a mechanism will be called a plan. As an endogenously determined plan, a core plan is a descriptive solution concept. It is not designed to fulfill any normative criterion. Indeed, some of the work of another paper will be cited later in the present section to clarify that a core plan is not (in general) ex post Pareto optimal (see the second paragraph that follows the statement of Definition 2.5).

The firm is divided into finitely many divisions. The goods, services, 
and resources that are used, produced, bought or sold by the divisions are called commodities. The commodities are classified into two categories: those $k_{m}$ commodities, called market commodities, that are bought or sold by the divisions, and those $k_{n}$ commodities, called nonmarket commodities, that are owned or produced by the firm and used internally. Nonmarket commodities can be multilaterally exchanged among the divisions. An important resource that is not considered a commodity here is the physical plant of each division; the present paper focuses on the situation in which plants are fixed for the division, thus are neither market commodities nor nonmarket commodities (divisions cannot sell them in the market nor can exchange them among themselves). ${ }^{3}$

Let $I$ be the set of divisions. Let $K_{m}$ be the set of market commodities, and let $K_{n}$ be the set of nonmarket commodities. The number of elements in set $K_{m}$ (in set $K_{n}$, resp.) is then $k_{m}\left(k_{n}\right.$, resp.). The set of commodities is defined ${ }^{4}$ as $K:=K_{m} \cup K_{n}$. Let $k:=k_{m}+k_{n}$, the number of elements in set $K$.

Division i's technology is embodied in its assets, human and physical; this is the phenomenon called the asset specificity. The know-how developed in the division represents the quality of its human resources, and is put into effect in production by installing specialized facilities. The benefit to the firm from internal use of these human resources and specialized facilities is much higher than their sale value in the market, so the firm makes every effort to keep them, that is, these assets are nonmarket commodities. A state of $i$ 's technology is, therefore, identified with a state of $i$ 's initially endowed nonmarket commodities. Division i's technology takes several possible states. These states are called here $i$ 's possible types. At a certain stage of production, $i$ 's true type becomes $i$ 's private information; that is, division $i$ knows its own type but not the others'.

To hold a patent, for example, is a particular way to use know-how. However, patented know-how is already public information. In the present paper, initially patented know-how does not distinguish states (qualities) of human resources. It may distinguish states (specificities) of physical facilities; the point is whether or not the physical facilities are appropriately specified for use of the patented know-how.

\footnotetext{
${ }^{3}$ See footnote 1 above.

${ }^{4}$ The notation " $a:=b$ " means " $a$ is equal to $b$ by definition."
} 
In the present paper the quantity of a nonmarket human resource $a$ is defined as

[the actual physical quantity] $\times$ [its efficiency (degree of specialization)],

so the amount of $a$ that division $i$ initially holds depends on $i$ 's type. By definition of embodied technology, the nonmarket non-human commodity bundle that $i$ initially holds depends on $i$ 's type. Thus, there is a 1-1 correspondence between $i$ 's type space and the range of its initially endowed nonmarket commodity vector.

Division $i$ 's state of technology, together with a condition on its noncommodity assets (such as $i$ 's plant), determines feasibility of input-output vectors in production. The feasibility is described by the production set concept (to be specified shortly).

A subset $S$ of $I$ is called a coalition of divisions. If the members of coalition $S$ fully pool their private information, they share the information structure generated by their private information. In reality, however, a member may not want to pass on to the others (a part of) its private information, and even if it does, the others may not take it as a truthful revelation of its type. The present paper addresses how private information is processed through coalitional choice of actions.

Formally, let $\mathcal{I}$ be the family of all nonempty coalitions of $I ; \mathcal{I}:=2^{I} \backslash\{\emptyset\}$. Let $T_{i}$ be the set of possible types of division $i$, which is assumed to be finite. Coalition $S$ 's type-profile space is given as $T_{S}:=\prod_{i \in S} T_{i}, S \in \mathcal{I}$. Set for simplicity, $T:=T_{I}$. Division $i$ 's private information structure is then defined as the algebra $\mathcal{T}_{i}$ on $T$ generated by the cylinder-sets, $\left\{\left\{t_{i}\right\} \times T_{I \backslash\{i\}} \mid t_{i} \in\right.$ $\left.T_{i}\right\}$. The information structure of $S$ generated by the members' private information is $\mathcal{T}_{S}:=\vee_{i \in S} \mathcal{T}_{i}$. The present paper addresses which subalgebra of $\mathcal{T}_{S}$ is endogenously determined through coalitional choice of actions.

Each division $i$ knows all the objective ex ante probabilities $\pi_{j}$ on $T_{j}$, $j \in I$. The divisions' types are assumed to be statistically independent. Thus the probability $\pi(t)$ of type profile is given by $\pi(t):=\prod_{i \in I} \pi_{i}\left(t_{i}\right)$. It is postulated $^{5}$ that $\pi \gg 0$.

A net output plan of division $i$ is a function $y_{i}: T \rightarrow \mathbf{R}^{k}$, which assigns to each type-profile $t$ a net output contingent upon $t, y_{i}(t):=\left(m_{i}(t), n_{i}(t)\right)$; the

\footnotetext{
${ }^{5}$ For two vectors $\pi$ and $\pi^{\prime}, \pi \geq \pi^{\prime}$ means $\pi(t) \geq \pi^{\prime}(t)$ for all $t, \pi>\pi^{\prime}$ means [ $\pi \geq \pi^{\prime}$ and $\left.\pi \neq \pi^{\prime}\right]$, and $\pi \gg \pi^{\prime}$ means $\pi(t)>\pi^{\prime}(t)$ for all $t$.
} 
subvectors $m_{i}(t)\left(\in \mathbf{R}^{k_{m}}\right)$ and $n_{i}(t)\left(\in \mathbf{R}^{k_{n}}\right)$ correspond to the market and nonmarket commodities, respectively. The usual sign convention is adopted here, so a coordinate of $y_{i}(t)$ is positive or negative according to whether the corresponding commodity is a net output or a net input of division $i$. The production set $Y_{i}\left(\subset \mathrm{R}^{k|T|}\right)$ is the set of all technologically feasible net output plans of $i$, where $|T|$ is the cardinality of $T$. Define $Y_{S}:=\prod_{i \in S} Y_{i}$, and $y_{S}:=\left(y_{i}\right)_{i \in S}$. Function $y_{S}$ is a net output plan of coalition $S$.

Two comments on the production sets are in order. First, to take the individual production sets $Y_{i}$ as given data, and derive the coalitional total production sets by $Y(S):=\sum_{i \in S} Y_{i}$ presupposes that there are no external economies or diseconomies. On the other hand, one may adopt a more general approach in which coalitional total production sets $Y(S), S \in \mathcal{I}$, are given data; in this case, external economies can be formulated as $Y(S) \supset$ $\sum_{i \in S} Y(\{i\})$. Although the main results of the present paper (Theorems 3.1, 4.2 and 5.2) are established for the non-externality case, generalizations to the externality case turn out to be straightforward. (See Remarks 3.2 and 4.4.)

Second, a specific instance of a production set is given by $Y_{i}=\prod_{t \in T} Y_{i}(t)$, $Y_{i}(t) \subset \mathbf{R}^{k}$. Here, $Y_{i}(t)$ is a $t$-contingent production set of division $i$. In this case, given type-profile $\bar{t},\left\{\bar{m}_{i}(t)\right\}_{t \in T \backslash \bar{t}}$ and $\bar{n}_{i}$, the production possibility set for market commodities at $\bar{t}$,

$$
\left\{m_{i}(\bar{t}) \in \mathbf{R}^{k_{m}} \mid\left(m_{i}(\bar{t}),\left\{\bar{m}_{i}(t)\right\}_{t \in T \backslash \bar{t}}, \bar{n}_{i}\right) \in Y_{i}\right\},
$$

is determined only by $\bar{n}_{i}(\bar{t})$, and is independent of net outputs $\bar{y}_{i}(t)$ 's chosen at the other type-profiles $t \neq \bar{t}$. The present general formulation, on the other hand, allows for the possibility that the production possibility set for market commodities at $\bar{t}$ depends on the entire menu of nonmarket net outputs across all type-profiles, $\left\{\bar{n}_{i}(t)\right\}_{t \in T}$, as well as on $\left\{\bar{m}_{i}(t)\right\}_{t \neq \bar{i}}$. The need for this general formulation arises if, for the production of net market output plan $m_{i}$, an adjustment of specific effort and/or non-commodity resources is required in addition to the choice of a net nonmarket output plan $n_{i}$, and different adjustments of effort/resources are required for different $m_{i}$ 's. This dependence (that is, the dependence of the production possibility set for market commodities at each $t$ on the entire nonmarket net output plan $n_{i}$ ) turns out to be crucial for the existence result in the case of increasing returns to scale (Theorem 4.2). (See Remarks 3.3, 4.5 and 5.3.) 
Given division $i$ 's type $t_{i} \in T_{i}$, the vector $r_{i}\left(t_{i}\right)\left(\in \mathbf{R}^{k_{n}}\right)$ of initial resources describes the quantities of nonmarket commodities that are assigned to (or owned by) division $i$ contingent upon $t_{i}$ before the production process.

A net output will be sold in the market under price vector $p\left(\in \mathbf{R}^{k_{m}}\right)$. The profit ${ }^{6}$ that division $i$ brings in to the firm by selling its net output of market commodities $m_{i}(t)$ is the inner product $p \cdot m_{i}(t)$. In the present partial equilibrium analysis, the price vector $p$ is exogenously given. It is postulated that $p \gg 0$.

In reality, however, there are likely very few $\mathrm{M}$-form firms that are pricetakers. In order to drop this perfect competition assumption on the markets, one may formally take each value $p_{a}$ to be equal to $1, a \in K_{m}$, and re-interpret the $a$-component of vector $m_{i}(t)$ as the profit, rather than the quantity, that the firm makes in the ath industry (so that $p \cdot m_{i}(t)$ is $i$ 's profit). According to this interpretation, the market prices made by the firm are hidden behind the model, and the downward sloping demand for outputs and upward sloping supply of inputs are subsumed in the divisional production sets. Of course, this can give rise to production externalities if different divisions purchase the same inputs or sell the same outputs. The coalitional total-production-set approach (see Remarks 3.2, 4.4) takes into account of such externalities. It should be pointed out, however, that to examine assumptions on the output demand functions and the input supply functions within this re-interpreted framework is outside the scope of the present paper.

DEFINITION 2.1 A profit-center game with incomplete information is a list of exogenously given data, $\mathcal{D}:=\left(\left\{T_{i}, \pi_{i}\right\}_{i \in I},\left\{Y_{i}, r_{i}(\cdot)\right\}_{i \in I}, p\right)$, of:

$T_{i}$, set of possible types of division $i$;

$\pi_{i}$, ex ante probability on $T_{i}$;

$Y_{i}$, production set of division $i$;

$r_{i}: T_{i} \rightarrow \mathbf{R}^{k_{n}}$, resource function of division $i$; and

$p$, price vector of the market commodities, such that $\pi_{i} \gg 0$ for all $i \in I$, and $p \gg 0$.

Given a type-profile $t \in T$, a profit imputation of coalition $S$ is a vector $x_{S}(t):=\left(x_{i}(t)\right)_{i \in S}$ whose $i$ th coordinate is to be interpreted as the accounting

\footnotetext{
${ }^{6}$ The present definition of profit is different from the neoclassical definition of profit, in that it need not reflect the cost of resources, such as capital, that are not under the control of the divisions.
} 
profit attributed to division $i$. The incentive of division $i$ is to claim as high a profit as possible for each $t$. A profit imputation plan of coalition $S$ is a function $x_{S}: T \rightarrow \mathbf{R}^{S}, t \mapsto x_{S}(t)$.

The ex ante stage is defined as the stage in which no division knows the true type of any division. The interim stage is defined as the stage in which each division in coalition $S$ knows its own true type and possibly has some information about the true types of some other divisions in $S$. The ex post stage is the stage in which each division knows the true type-profile in $T$.

Here is a rough outline of the scenario of how a production process in coalition $S$ takes place (this paragraph and the next two paragraphs). Suppose that the members of coalition $S$ have agreed on a pair $\left(x_{S}, y_{S}\right)$ of a profit imputation plan and a net output plan at the ex ante stage. A pair $\left(x_{S}, y_{S}\right)$ will henceforth be called a plan. An agreement on a plan, once made, will remain binding throughout the production periods. The production process takes place for two periods of the interim stage: The first interim period is for the divisions' simultaneous decisions about initial investment, that is, about setting up their manufacturing processes. The second interim period is for subsequent decisions about actual manufacturing, that is, about choice of an input-output vector, and for imputation of the profit that is made by sale/purchase of the market commodities. There are $k_{1}$ commodities, market or nonmarket, whose supply/demand constitutes the setup of a manufacturing process in the first period. The other $k_{2}$ commodities, also market or nonmarket, are supplied/demanded in the second period as outputs/inputs, $k_{2}:=k-k_{1}$.

At the beginning of the first period, Nature reveals to division $i$ that its true type is $\bar{t}_{i}$; this is $i$ 's private information at that time. Based upon this information, division $i$ chooses in the first period the $k_{1}$-dimensional subvector of an initial net investment according to the agreement. This subvector is observed by every member of the coalition, so assuming that choices of these subvectors are made according to the true types $\bar{t}_{i}, i \in S$, every division knows by the end of the first period the realization of the event defined as the set of those type-profiles in $T_{S}$ which give rise to the observed subvectors. In the second period, division $i$ uses this new information (and again its knowledge about its true type $\bar{t}_{i}$ ) to choose the $k_{2}$-dimensional subvector of input-output and receive its profit imputation. In short, a feasible plan has to reflect the various pieces of information made available to each division during the production process (the information-pooling rule; see Postulate 
2.2 below), in addition to the technological and resource constraints.

Before the production process, that is, in the ex ante stage, all feasible plans $\left(x_{S}, y_{S}\right), S \in \mathcal{I}$, are negotiated about by the divisions. The preceding paragraph outlined that as a part of the feasibility constraint, these plans have to satisfy the information-pooling rule. Plans should also be designed carefully enough so that they guarantee their truthful execution (Bayesian incentive compatibility; see Postulate 2.3 below). One may imagine the negotiation process as a succession of coalition formations, each coalition improving upon the plan of the preceding coalition. The Bayesian incentive compatibility may be so stringent a condition that there may not be a feasible plan that satisfies it and at the same time encounters no "blocking" coalition. To resolve this difficulty, the firm's headquarters is given the role of an insurer (see Postulate 2.4 below). Eventually, the grand coalition $I$ is formed, and the divisions are settled at a feasible and Bayesian incentive-compatible plan $\left(x_{I}^{*}, y_{I}^{*}\right)$ that no coalition can improve upon. The plan $\left(x_{I}^{*}, y_{I}^{*}\right)$ is sustained throughout the rest of the negotiation period of the ex ante stage. It is this self-enforcing plan that is agreed upon in the firm.

To start the precise description of the scenario, denote by $F_{S}$ the set of all technologically attainable plans of a coalition $S$, that is, the set of all functions $\left(x_{S}, y_{S}\right): T_{S} \rightarrow \mathbf{R}^{(1+k)|S|}$ such that $y_{S}$ is technologically feasible, i.e., $y_{S} \in Y_{S}$, and such that the total resource constraint is satisfied within $S$, i.e.,

$$
\forall t_{S} \in T_{S}: \sum_{i \in S}\left(\begin{array}{c}
x_{i}\left(t_{S}\right) \\
0
\end{array}\right) \leq \sum_{i \in S}\left(\begin{array}{c}
p \cdot m_{i}\left(t_{S}\right) \\
n_{i}\left(t_{S}\right)+r_{i}\left(t_{i}\right)
\end{array}\right) .
$$

Notice that while a plan is in general defined as a function of $t$, the technological attainability imposes the condition that it be a function only of $t_{S}$; this condition reflects the fact that the maximal information possibly available to each member of $S$ is the algebra $\mathcal{T}_{S}$ on $T$. Dependence of a plan only on $t_{S}$ is re-stated as its $\mathcal{T}_{S}$-measurability as a function on $T$. In order to simplify notation, however, one may write $\left(x_{S}(t), y_{S}(t)\right)$ instead of $\left(x_{S}\left(t_{S}\right), y_{S}\left(t_{S}\right)\right)$ throughout this paper; no confusion arises. Notice also that negative imputation is allowed. ${ }^{7}$

Let $K_{1}$ be the index set for the $k_{1}$ commodities that are produced/used in the first period as an initial investment, and let $K_{2}$ be the index set for the $k_{2}$

\footnotetext{
${ }^{7}$ In the main results of this paper (Theorems 3.1, 4.2 and 5.2), the existence of an equilibrium plan $\left(x_{I}^{*}, y_{I}^{*}\right)$, called a core plan, for which $\forall t: \forall i: x_{i}^{*}(t) \geq 0$ is asserted.
} 
commodities that are produced/used in the second period as outputs/inputs. The family $\left\{K_{1}, K_{2}\right\}$ is a partition of $K$, possibly different from partition $\left\{K_{n}, K_{m}\right\}$. A net output plan $y_{i}$ may be written as

$$
y_{i}=\left(\begin{array}{c}
y_{1 i} \\
y_{2 i}
\end{array}\right),
$$

where

$$
\begin{array}{ll}
y_{1 i}: & T \rightarrow \mathbf{R}^{k_{1}}, \quad t \mapsto y_{1 i}(t), \\
y_{2 i}: & T \rightarrow \mathbf{R}^{k_{2}}, \quad t \mapsto y_{2 i}(t),
\end{array}
$$

so the components of $y_{1 i}(t)$ (of $y_{2 i}(t)$, resp.) correspond to $K_{1}\left(K_{2}\right.$, resp.). Define $y_{1 S}:=\left(y_{1 i}\right)_{i \in S}, y_{2 S}:=\left(y_{2 i}\right)_{i \in S}$. By the information-pooling rule (to be precisely postulated shortly), $y_{1 i}$ is actually a function only of $t_{i}$, and $y_{2 i}$ is a function only of $t_{i}$ and the information pooled through $y_{1 S}$.

Suppose coalition $S$ is to form, so the members consider a plan $\left(x_{S}, y_{S}\right)$. The private information structure that division $i$ holds at the beginning of the first period is $\mathcal{T}_{i}$, so $y_{1 i}$ has to depend only upon $t_{i}$ (that is, it has to be $\mathcal{T}_{i}$-measurable). Let $\mathcal{A}\left(y_{1 j}\right)$ be the algebra on $T$ generated by function $y_{1 j}: T \rightarrow \mathbf{R}^{k_{1}}$, that is, the smallest algebra on $T$ that contains the family of sets $\left\{\left(y_{1 j}\right)^{-1}\left(\eta_{1 j}\right) \mid \eta_{1 j} \in \mathbf{R}^{k_{1}}\right\}$. Assuming that choices of $y_{1 i}\left(t_{i}\right)$ 's are made according to the true types, the information structure $\vee_{j \in S} \mathcal{A}\left(y_{1 j}\right)$ becomes a common knowledge in coalition $S$ at the beginning of the second period. Define

$$
\hat{\mathcal{T}}_{i}\left(y_{1 S}\right):=\mathcal{T}_{i} \vee\left(\vee_{j \in S} \mathcal{A}\left(y_{1 j}\right)\right) .
$$

The members can design $y_{S}$ at the ex ante stage, so that each $\left(x_{i}, y_{2 i}\right)$ is $\hat{T}_{i}\left(y_{1 S}\right)$-measurable. Thus, one can make the following postulate:

POSTULATE 2.2 (Information-Pooling Rule) The members of coalition $S$ can design only those plans $\left(x_{S}, y_{S}\right)$ such that for each $i \in S$ it follows that

(i) division i's plan for the first interim period is a function only of its type, i.e., $y_{1 i}$ is $\mathcal{T}_{i}$-measurable,

(ii) division $i$ 's plan for the second interim period is a function only of its type and the pooled information of its own coalition, i.e., $\left(x_{i}, y_{2 i}\right)$ is $\hat{\mathcal{T}}_{i}\left(y_{1 S}\right)$ measurable. 
In the light of the information-pooling rule, define $F_{S}^{\prime}$ to be the set of allowable plans for $S$, i.e.,

$$
F_{S}^{\prime}:=\left\{\begin{array}{l|l}
\left(x_{S}, y_{S}\right) \in F_{S} & \begin{array}{l}
\forall i \in S: \\
y_{1 i} \text { is } \mathcal{T}_{i} \text {-measurable, } \\
\left(x_{i}, y_{2 i}\right) \text { is } \hat{\mathcal{T}}_{i}\left(y_{1 S}\right) \text {-measurable }
\end{array}
\end{array}\right\} .
$$

In order for the present information-pooling rule to work, the members of coalition $S$ need to have the common knowledge that each division has the incentive to make a choice in the first period according to its true type. After all, the agreement will not be enforced, if some member has the strict incentive to make a choice with false pretension about its true type either during the first period or during the second period. If the members of $S$ foresee at the time of designing a plan that a particular plan $\left(x_{S}, y_{S}\right)$ may later induce such false pretension, they do not agree on the plan $\left(x_{S}, y_{S}\right)$. They will consider only those plans that are Bayesian incentive-compatible.

The present Bayesian incentive compatibility concept extends the original definition of d'Aspremont and Gerard-Varet (1979) to the situations that are consistent with the information-pooling rule (Postulate 2.2). Suppose the members of coalition $S$ agree upon plan $\left(x_{S}, y_{S}\right) \in F_{S}^{\prime}$ in the ex ante stage. Suppose division $i$ 's true type is $\bar{t}_{i}, i \in S$. In the first interim period, while division $i$ is required to take action $y_{1 i}\left(\bar{t}_{i}\right)$, it actually has liberty of taking any action from $y_{1 i}\left(T_{i}\right)$, since its true type is private. Let $\tilde{\eta}_{1 i} \in y_{1 i}\left(T_{i}\right)$ be the action it takes. The other divisions then infer that $i$ 's true type must be in the set $y_{1 i}^{-1}\left(\tilde{\eta}_{1 i}\right) \subset T_{i}$. If $\tilde{\eta}_{1 i}$ is a false action, that is, if $\tilde{\eta}_{1 i} \neq y_{1 i}\left(\bar{t}_{i}\right)$, division $i$ is giving the others false information about its true type. Assuming that the other divisions are honest, division $i$ in turn receives the information that division $j$ 's true type is in the set $y_{1 j}^{-1}\left(y_{1 j}\left(\bar{t}_{j}\right)\right), j \in S \backslash\{i\}$. In the second interim period, division $i$ can choose any action as long as its possible misrepresentation in the first interim period is not caught with this secondround action. Here, the other divisions have been led to believe that the true type-profile must be in the event

$$
\tilde{T}:=y_{1 j}^{-1}\left(\tilde{\eta}_{1 i}\right) \times \prod_{j \in S \backslash\{i\}} y_{1 j}^{-1}\left(y_{1 j}\left(\bar{t}_{j}\right)\right) \subset T .
$$

Division $i$ will not be caught in the second period, therefore, as long as it chooses its action $\left(\tilde{\eta}_{2 i}, \tilde{\xi}_{i}\right)$ from the set $y_{2 i}(\tilde{T}) \times x_{i}(\tilde{T})$. Coalition $S$ 's plan 
$\left(x_{S}, y_{S}\right)$ is called Bayesian incentive-compatible, if division $i$ cannot receive a higher benefit from any such pair of actions $\left(\tilde{\eta}_{1 i},\left(\tilde{\eta}_{2 i}, \tilde{\xi}_{i}\right)\right)$ than from the honest actions. A precise definition, which is technically more involved, will be given at the outset of Section 6 .

POSTULATE 2.3 (Bayesian Incentive Compatibility) The members of coalition $S$ can design only Bayesian incentive-compatible plans.

In the light of the Bayesian incentive compatibility, define $F_{S}^{\prime \prime}$ to be the set of allowable, Bayesian incentive-compatible plans for $S$, i.e.,

$$
F_{S}^{\prime \prime}:=\left\{\left(x_{S}, y_{S}\right) \in F_{S}^{\prime} \mid\left(x_{S}, y_{S}\right) \text { is Bayesian incentive-compatible }\right\} .
$$

Facts 6.1 and 6.2 in Section 6 establish necessary and sufficient conditions for an allowable plan $\left(x_{S}, y_{S}\right) \in F_{S}^{\prime}$ to be Bayesian incentive-compatible. For any algebra $\mathcal{B}$ on $T$, let $E\left(x_{i} \mid \mathcal{B}\right): T \rightarrow \mathbf{R}$ be the conditional expectation of $x_{i}$ given $\mathcal{B}$, defined as

$$
E\left(x_{i} \mid \mathcal{B}\right)(t):=\frac{1}{\pi(B(t))} \sum_{s \in B(t)} x_{i}(s) \pi(s),
$$

where $B(t)$ is the minimal element of $\mathcal{B}$ that contains $t$. One condition equivalent to the Bayesian incentive compatibility is

$$
\begin{aligned}
& \forall i \in S: \forall t_{i}, t_{i}^{\prime} \in T_{i}: \forall \bar{t}_{I \backslash\{i\}} \in T_{I \backslash\{i\}}: \\
& \quad E\left(x_{i} \mid \hat{\mathcal{T}}_{i}\left(y_{1 S}\right)\right)\left(t_{i}, \bar{t}_{I \backslash\{i\}}\right)=E\left(x_{i} \mid \hat{\mathcal{T}}_{i}\left(y_{1 S}\right)\right)\left(t_{i}^{\prime}, \bar{t}_{I \backslash\{i\}}\right),
\end{aligned}
$$

which means that for any division $i \in S$ and for any minimal element $A$ ( $\subset T_{T \backslash\{i\}}$ ) of the information structure on the other divisions' types available to $i$ after the first interim stage (an minimal element of $\mathcal{A}\left(y_{1, S \backslash\{i\}}\right)$ ), the conditional expectation of $x_{i}$ given $\left\{t_{i}\right\} \times A$ is independent of $t_{i}$. This condition is not surprising, in view of the fact that the von Neumann-Morgenstern utility function of each division $i$ is $i$ 's profit-imputation, in particular, it is type-independent.

The set of strategies $F_{S}^{\prime \prime}$ summarizes what coalition $S$ can do by collaboration of its members. One may study the following game: Start with any plan $\left(x_{I}^{0}, y_{I}^{0}\right) \in F_{I}^{\prime \prime}$. If the members of some coalition $S_{1} \in \mathcal{I}$ can improve upon 
it, ${ }^{8}$ the new coalition $S_{1}$ will indeed be formed with its dominating strategy bundle $\left(x_{S_{1}}^{1}, y_{S_{1}}^{1}\right) \in F_{S_{1}}^{\prime \prime}$. Choose any $\left(x_{\Gamma \backslash S_{1}}^{1}, y_{\Gamma \backslash S_{1}}^{1}\right) \in F_{I \backslash S_{1}}^{\prime \prime}$. If the members of some other coalition $S_{2} \in \mathcal{I}$ can improve upon $\left(x_{I}^{1}, y_{I}^{1}\right)$, the new coalition $S_{2}$ will be formed with its dominating strategy bundle $\left(x_{S_{2}}^{2}, y_{S_{2}}^{2}\right) \in F_{S_{2}}^{\prime \prime}$. In this way, there will be successive formations of coalitions, $S_{1}, S_{2}, \cdots$, in the $e x$ ante period. The sequence of coalition formations may continue only until a particular strategy bundle $\left(x_{I}^{\tau}, y_{I}^{\tau}\right)$ is reached in the $\tau$ th round, upon which no coalition can improve; in which case $\left(x_{I}^{\tau}, y_{I}^{\tau}\right)$ is called a core plan, and the divisions will sign it as their self-enforcing contracts. The sequence of coalition formations may continue infinitely, so that there is no possibility for agreement; in which case the headquarters may step in to modify the game so that the modified game has a core plan.

At present, the present authors do not know whether the game based upon the sets $F_{S}^{\prime \prime}, S \in \mathcal{I}$, really has a core plan. While this is left as an important open problem, this paper studies a modified game and establishes conditions for the existence of its core plan. The modified game introduces the possibility that the headquarters can play a role of insurer to the divisions.

A plan $\left(x_{S}, y_{S}\right)$ is called weakly Bayesian incentive-compatible if for all $i \in S$,

$$
\forall t_{i}, t_{i}^{\prime} \in T_{i}: E\left(x_{i} \mid t_{i}\right) \geq E\left(x_{i}\left(t_{i}^{\prime}, \cdot\right) \mid t_{i}\right),
$$

where $E\left(x_{i} \mid t_{i}\right):=E\left(x_{i} \mid \mathcal{T}_{i}\right)(t)$. It is straightforward (see Fact 6.2 (ii)) that for a weakly Bayesian incentive-compatible plan $\left(x_{S}, y_{S}\right)$, the conditional expectation $E\left(x_{i} \mid t_{i}\right)$ is independent of $t_{i}$, that is, $E\left(x_{i} \mid \mathcal{T}_{i}\right)$ is a constant function. This fact motivates the following formulation of the role of the headquarters as an insurer:

POSTULATE 2.4 (Headquarters' Insurability) Let $\left(x_{I}, y_{I}\right)$ be a technologically attainable plan of the grand coalition such that it satisfies the information-pooling rule, and $E\left(x_{i} \mid \mathcal{T}_{i}\right)$ is a constant function for each $i \in I$. Then the plan $\left(\left(E\left(x_{i} \mid \mathcal{T}_{i}\right)\right)_{i \in I}, y_{I}\right)$ is available to the grand coalition $I$.

Being an insurer is the only role that the headquarters plays in this game, in addition to participating in the coalitional design of a plan as one of the divisions. By Postulate 2.4, division $i$ can receive the accounting profit according to the constant profit imputation plan $E\left(x_{i} \mid \mathcal{T}_{i}\right)$. This is justified

\footnotetext{
${ }^{8}$ The precise content of "improve upon" will be clarified in the following Definition 2.5 .
} 
if the headquarters is risk-neutral. Moreover, this is an easy task for the headquarters, because it does not have to know the true type of division $i$ (the need for insurance occurs only when $E\left(x_{i} \mid t_{i}\right)$ is the same for all $t_{i}$ ). Postulate 2.4 does not reduce the model to a static game. Indeed, while the profit imputation plan for the grand coalition, $\left(E\left(x_{i} \mid \mathcal{T}_{i}\right)\right)_{i \in I}$, stated in the postulate has a static flavor as a constant function, it is made possible by non-constant net output function $y_{I}$, and the latter is subject to the information-pooling rule. One needs to study how the private information is processed in accordance with this rule.

Postulate 2.4 (headquarters' insurability) enlarges the feasible-strategy set of the grand coalition, in such a way that the newly introduced strategies are consistent with the other postulates: Postulate 2.2 (informationpooling rule) and Postulate 2.3 (Bayesian incentive compatibility). Indeed, let $\left(x_{I}, y_{I}\right)$ be a technologically attainable plan of the grand coalition such that $y_{I}$ satisfies the information-pooling rule, and $E\left(x_{i} \mid \mathcal{T}_{i}\right)$ is a constant function for each $i \in I$. Then the plan $\left(\left(E\left(x_{i} \mid \mathcal{T}_{i}\right)\right)_{i \in I}, y_{I}\right)$ is Bayesian incentive compatible.

The headquarters' insurability is frequently observed in reality. If division $i$ is performing unexpectedly poorly, the headquarters ends up paying more accounting profit to $i$ than the amount that $i$ contributes to the firm, which is a form of insurance. One the other hand, insurance among subordinate divisions rarely occurs; it occurs only through the headquarters.

Formally, let $H_{I}$ be the set of all plans $\left(x_{I}, y_{I}\right)$ for the grand coalition $I$ such that $x_{I}$ is a constant function, and such that there exists $x_{I}^{\prime}: T \rightarrow \mathbf{R}^{|I|}$ for which $\left(x_{I}^{\prime}, y_{I}\right) \in F_{I}^{\prime}$ and $E\left(x_{i}^{\prime} \mid \mathcal{T}_{i}\right)=x_{i}$ for every $i \in I$. Notice that every member of $H_{I}$ is Bayesian incentive-compatible. In the light of the headquarters' insurability, define:

$$
\hat{F}_{S}:=\left\{\begin{array}{l}
F_{S}^{\prime \prime}, \text { if } S \neq I \\
F_{I}^{\prime \prime} \cup H_{I}, \text { if } S=I .
\end{array}\right.
$$

The set $\hat{F}_{S}$ is the set of all technologically attainable plans of coalition $S$ for insurable plans - only in the case $S=I$ ) that are consistent with the three postulates (Postulates $2.2,2.3,2.4)$. Plan $\left(x_{S}, y_{S}\right)$ is a candidate for coalition $S$ 's agreement, iff $\left(x_{S}, y_{S}\right) \in \hat{F}_{S}$.

Suppose the grand coalition $I$ is considering a possible agreement $\left(x_{I}^{*}, y_{I}^{*}\right) \in$ $\hat{F}_{I}$. Coalition $S$ will improve upon $\left(x_{I}^{*}, y_{I}^{*}\right)$, if it finds a plan $\left(x_{S}, y_{S}\right) \in \hat{F}_{S}$ 
such that, for each member $i$, the conditional expectation of $x_{i}$ given $\hat{T}_{i}\left(y_{1 S}\right)$ dominates the conditional expectation of $x_{i}^{*}$ given $\hat{\mathcal{T}}_{i}\left(y_{1 S}^{*}\right)$. If plan $\left(x_{I}^{*}, y_{I}^{*}\right)$ is not improved upon by any coalition, it becomes a self-enforcing agreement. A core plan is a plan of the grand coalition which is feasible and which cannot be improved upon by any coalition:

DEFINITION 2.5 A core plan of profit-center game with incomplete information $\mathcal{D}$ is a plan $\left(x_{I}^{*}, y_{I}^{*}\right)$ of the grand coalition $I$ such that (i) $\left(x_{I}^{*}, y_{I}^{*}\right) \in \hat{F}_{I}$; and

(ii) it is not true that there exist $S \in \mathcal{I}$ and $\left(x_{S}, y_{S}\right) \in \hat{F}_{S}$ such that for all $i \in S$,

$$
\begin{array}{ll}
\forall t \in T: & E\left(x_{i} \mid \hat{\mathcal{T}}_{i}\left(y_{1 S}\right)\right)(t) \geq E\left(x_{i}^{*} \mid \hat{\mathcal{T}}_{i}\left(y_{1 I}^{*}\right)\right)(t), \quad \text { and } \\
\exists t \in T: & E\left(x_{i} \mid \hat{\mathcal{T}}_{i}\left(y_{1 S}\right)\right)(t)>E\left(x_{i}^{*} \mid \hat{\mathcal{T}}_{i}\left(y_{1 I}^{*}\right)\right)(t) .
\end{array}
$$

A core plan $\left(x_{I}^{*}, y_{I}^{*}\right)$ is called full-information revealing, if for every $i \in I$, $y_{1 i}^{*}$ is $1-1$ on $T_{i}$. In this case, the algebra $\hat{\mathcal{T}}_{i}\left(y_{1 I}^{*}\right)$ becomes the finest algebra $2^{T}$. Full information is revealed during the period of setting up manufacturing processes, and the subsequent choice of input/output vectors is based on the full information.

A core plan is a descriptive solution concept. Ichiishi and Sertel (forthcoming, Example 4.4) shows that a full-information revealing core is not ex post Pareto optimal, and this non-optimality comes from Postulate 2.3 (Bayesian incentive compatibility) and Postulate 2.2 (information-pooling rule). The role of Bayesian incentive compatibility in yielding inefficiency is well-known in the principal-agent literature. The information-pooling rule also creates inefficiency, because the first interim period strategy $y_{1 i}$ is subject to the constraint of $\mathcal{T}_{i}$-measurability.

For commodity $a \in K_{n}$, denote by $r_{i}\left(t_{i}, a\right)$ the ath component of $r_{i}\left(t_{i}\right)$. A nonmarket intermediate commodity is a commodity $a \in K_{n}$, such that $r_{i}\left(t_{i}, a\right)=0$ for all $i \in I$ and $t \in T$, but such that the firm produces it (at some division, say $i$ ) and internally uses it (at another division, say $j$ ). Since there is no market price for this commodity, division $j$ does not know how much transfer payment to make to division $i$ for its use. However, a core imputation plan $x_{I}^{*}$ provides an answer.

To state the basic assumption of the present paper, define

$$
K_{1 n}:=K_{1} \cap K_{n}, k_{1 n}:=\left|K_{1 n}\right|,
$$


and define also $K_{2 n}, K_{1 m}, K_{2 m}, k_{2 n}, k_{1 m}$ and $k_{2 m}$ analogously. The initial resource function $r_{i}$ may be written as

$$
r_{i}=\left(\begin{array}{c}
r_{1 i} \\
r_{2 i}
\end{array}\right),
$$

where $r_{1 i}$ is a function from $T_{i}$ to $\mathbf{R}^{k_{1 n}}$, and the components of $r_{1 i}\left(t_{i}\right)$ correspond to $K_{1 n}$.

The following Assumption 2.6 imposes conditions on the production set of each division. In particular, (ii) says that zero production activity is possible; (iii) means free disposal; and (iv) means the impossibility of the Land of Cockaigne, that is, only a finite quantity of outputs is produced from a finite quantity of inputs.

\section{ASSUMPTION 2.6 (Basic Assumptions on the Production Sets)} For each $i$,

(i) the production set $Y_{i}$ is closed in $\mathrm{R}^{k|T|}$;

(ii) $0 \in Y_{i}$;

(iii) $Y_{i}-\mathbf{R}_{+}^{k|T|} \subset Y_{i}$;

(iv) for each $n_{i} \in \mathbf{R}^{k_{n}|T|}$, the production possibility set $\left\{m_{i} \in \mathbf{R}^{k_{m}|T|} \mid\right.$ $\left.\left(m_{i}, n_{i}\right) \in Y_{i}\right\}$ is bounded from above.

The following Assumption 2.7 imposes conditions on the resource function of each division. In particular, (i) says that there are nonmarket commodities which are used or produced in the first period. Condition (ii) says that $r_{1 i}$ takes different values for different types of division $i$; the fourth through the seventh paragraphs of the present section have given a detailed account of the necessity of this 1-1 property, when $i$ 's type is defined as a state of its technology embodied in its initial investment. Condition (iii) says that all resources are nonnegative.

ASSUMPTION 2.7 (Basic Assumptions on the Resource Functions) (i) $K_{1 n} \neq \emptyset$;

For each $i \in I$,

(ii) the function $r_{1 i}$ is $1-1$ on $T_{i}$;

(iii) $r_{i}\left(t_{i}\right) \geq 0$, for all $t_{i} \in T_{i}$. 
REMARK 2.8 In the present model, divisions spend one period for setting up manufacturing processes and another period for actual manufacturing. In reality, however, divisions repeat manufacturing in many subsequent periods, forecasting at each period the new market demand condition. So, one needs a model of one set-up period and arbitrarily many periods for repeated manufacturing. This task is, however, beyond the scope of the present paper.

\section{Convex Production Sets}

The first result of this paper is the following existence theorem for the neoclassical case of convex technology. The theorem is consistent with the presence of nonmarket intermediate commodities.

THEOREM 3.1 Let $\mathcal{D}$ be a profit-center game with incomplete information which satisfies Postulates 2.2-2.4 (information-pooling rule, Bayesian incentive compatibility, and headquarters' insurability) and Assumptions 2.6-2.7 (basic assumptions on the production sets, and on the resource functions). Assume moreover that the production set $Y_{i}$ is convex, for each $i \in I$. Then there exists a full-information revealing core plan of the game.

REMARK 3.2 Instead of the approach of the text in which $Y_{i}, i \in I$, are given primitive data, consider a more general approach in which coalitional total production sets $Y(S), S \in \mathcal{I}$, are given. Theorem 3.1 still holds true if each $Y(S)$ satisfies Assumptions 2.6-2.7, convexity, and the following balancedness condition: For any balanced subfamily $\mathcal{B}$ of $\mathcal{I}$ and for any associated balancing coefficients $\left\{\lambda_{S}\right\}_{S \in \mathcal{B}}$, it follows that $\sum_{S \in \mathcal{B}} \lambda_{S} Y(S) \subset Y(I)$. The balancedness condition makes explicit the extent to which external economies should prevail in the grand coalition. Notice that if $Y(S)=$ $\sum_{i \in S} Y_{i}$, then the balancedness condition is automatically satisfied.

REMARK 3.3 In the specific instance of a production set given by $Y_{i}=$ $\prod_{t \in T} Y_{i}(t)$, all the assumptions on $Y_{i}$ of Theorem 3.1 are satisfied, provided that each $Y_{i}(t)$ satisfies Assumptions 2.6-2.7 and convexity.

REMARK 3.4 Fix the data $\left(\left\{T_{i}, \pi_{i}\right\}_{i \in I},\left\{Y_{i}, r_{2 i}(\cdot)\right\}_{i \in I}, p\right)$ throughout this remark. Then, a profit-center game $\left(\left\{T_{i}, \pi_{i}\right\}_{i \in I},\left\{Y_{i}, r_{i}(\cdot)\right\}_{i \in I}, p\right)$ is identified 
with the function $r_{1 I}(\cdot)$. By varying $r_{1 I}(\cdot) \in \prod_{i \in I} \mathbf{R}_{+}^{k_{1 n}\left|T_{i}\right|}$, one obtains different profit-center games. Therefore, the set $\mathcal{G}:=\prod_{i \in I} \mathbf{R}_{+}^{k_{1 n}\left|T_{i}\right|}$, endowed with the relativized Euclidean topology, becomes the Baire space of profit-center games. It is easy to see that the subset

$$
\mathcal{G}_{0}:=\left\{r_{1 I} \in \mathcal{G} \mid \forall i \in I: r_{1 i} \text { is } 1-1 \text { on } T_{i}\right\}
$$

is open and dense in $\mathcal{G}$. So, without condition (ii) of Assumption 2.7, a generic existence theorem still holds true. The same remark applies also to Theorem 4.2.

\section{Distributive Total Production Set of the Grand Coalition}

This section presents an existence theorem for a particular technology which satisfies increasing returns to scale. The theorem, however, excludes nonmarket intermediate commodities. Nevertheless, nonmarket commodities held as the initial resources can be exchanged among the divisions, and a core imputation plan describes in part transfer payments for these exchanges.

Introduce a fictitious price vector, $q:=(q(t))_{t \in T}, q(t)=\left(q_{1}(t), q_{2}(t)\right) \in$ $\mathbf{R}_{+}^{k_{1 n}} \times \mathbf{R}_{+}^{k_{2 n}}$, for type-profile-contingent nonmarket commodities; this is to utilize a cost function concept in the analysis. Set $q(t):=(q(t, a))_{a \in K_{n}}$. One may choose the unit simplex,

$$
Q:=\left\{q: T \rightarrow \mathbf{R}_{+}^{k_{n}} \mid \sum_{t \in T} \sum_{a \in K_{n}} q(t, a)=1\right\},
$$

as the price domain.

The exclusion of nonmarket intermediate commodities means that all the nonmarket commodities are only used as inputs. Given a market net output plan $m: T \rightarrow \mathbf{R}^{k_{m}}$, therefore, one may define the input-requirement set as

$$
S_{Y_{I}}(m):=\left\{\begin{array}{l|l}
-n: T \rightarrow \mathbf{R}^{k_{n}} & \begin{array}{l}
(m, n) \leq \sum_{i \in I}\left(m_{i}, n_{i}\right), \\
\forall i \in I: \begin{array}{l}
\left(m_{i}, n_{i}\right) \in Y_{i}, \\
y_{1 i} \text { is } \mathcal{T}_{i} \text {-measurable }
\end{array}
\end{array}
\end{array}\right\} .
$$

A cost function is then defined as the function $g: Q \times \mathbf{R}^{k_{m}|T|} \rightarrow \mathbf{R}_{+}$which associates to each pair of a fictitious price vector and a market net output 
plan $(q, m)$ the minimal cost of using nonmarket commodities in order to produce $m$ under $q$. Formally, it is defined by

$$
g(q, m):=\inf \left\{-\sum_{t \in T} q(t) \cdot n(t) \mid-n \in S_{Y_{I}}(m)\right\} .
$$

The concept of weak Bayesian incentive compatibility was introduced earlier (in the second paragraph following Postulate 2.3). Another type of cost function which accommodates the weak Bayesian incentive compatibility, $c_{S}^{\text {wic }}: Q \times \stackrel{\circ}{\mathbf{R}}_{+} \rightarrow \mathbf{R}_{+}$, is now defined: In order to do so, choose any profit level $\eta$, and consider the set of all pairs of a profit imputation plan and a total net output plan $\left(x_{S}, \sum_{i \in S} y_{i}\right)$ such that (1) each $y_{i}$ is technologically feasible, (2) the total imputation $\sum_{i \in S} x_{i}(t)$ is met by the total profit $\sum_{i \in S} p \cdot m_{i}(t)$ for every possible type-profile $t$, and (3) the conditional expectations $E\left(x_{i} \mid t_{i}\right)$ of the imputations $x_{i}$ given $i$ 's private information $\mathcal{T}_{i}, i \in S$, are summed up at least to $\eta$ for any possible type-profile $t$. Formally, the set is given as:

$$
C_{S}(\eta):=\left\{\begin{array}{l|l}
\left(x_{S}, \sum_{i \in S} y_{i}\right) & \begin{array}{l}
y_{i} \in Y_{i}, \\
\forall t \in T: \sum_{i \in S} x_{i}(t) \leq \sum_{i \in S} p \cdot m_{i}(t), \\
\eta \leq \sum_{i \in S} \min _{t \in T} E\left(x_{i} \mid \mathcal{T}_{i}\right)(t), \\
\left(x_{S}, m_{S}\right) \text { is } \mathcal{T}_{S} \text {-measurable, } \\
\forall i \in S: y_{1 i} \text { is } \mathcal{T}_{i} \text {-measurable }
\end{array}
\end{array}\right\} .
$$

Define the mathematical programming problem,

$$
\begin{aligned}
& \text { Problem } P_{S}(q, \eta) \text { : Minimize }-\sum_{t \in T} q(t) \cdot n(t) \text {, } \\
& \text { subject to }\left(x_{S}, y\right) \in C_{S}(\eta) \text {. }
\end{aligned}
$$

The required cost function is the optimal value of Problem $P_{S}(q, \eta)$ :

$$
c_{S}^{w i c}(q, \eta):=\inf \left\{-\sum_{t \in T} q(t) \cdot n(t) \mid\left(x_{S}, y\right) \in C_{S}(\eta)\right\} .
$$

The remark at the end of this section briefly sketches why the function $c_{S}^{\text {wic }}$ takes into account the weak Bayesian incentive compatibility (see Remark 4.6). 
For a net output plan $y_{i}$ for which $y_{1 i}$ is $\mathcal{T}_{i}$-measurable, the cost is

$$
\begin{aligned}
-\sum_{t \in T} q(t) \cdot n_{i}(t)= & -\sum_{t_{i} \in T_{i}}\left\{\sum_{t_{T \backslash\{i\}} \in T_{T \backslash\{i\}}} q_{1}\left(t_{i}, t_{T \backslash\{i\}}\right)\right\} \cdot n_{1 i}\left(t_{i}\right) \\
& -\sum_{t \in T} q_{2}(t) \cdot n_{2 i}(t),
\end{aligned}
$$

so Problem $P_{S}(q, \eta)$ has an optimal solution, if

$$
\forall i \in S: \forall t_{i} \in T_{i}: \sum_{t_{I \backslash\{i\}} \in T_{T \backslash\{i\}}} q_{1}\left(t_{i}, t_{I \backslash\{i\}}\right) \gg 0,
$$

and

$$
\forall t \in T: q_{2}(t) \gg 0 .
$$

The same remark applies to the earlier minimization problem that defines $g(q, m)$. This fact motivates the following definitions:

$$
\begin{aligned}
Q_{+}:= & \bigcap_{i \in I t_{i} \in T_{i}}\left\{q \in Q \mid \sum_{t_{I \backslash\{i\}} \in T_{I \backslash\{i\}}} q_{1}\left(t_{i}, t_{I \backslash\{i\}}\right) \gg 0\right\} \\
& \bigcap \bigcap_{t \in T}\left\{q \in Q \mid q_{2}(t) \gg 0\right\}, \\
Q_{0}:= & Q \backslash Q_{+} .
\end{aligned}
$$

Problem $P_{S}(q, \eta)$ and the problem for $g(q, m)$ have optimal solutions, if $q \in$ $Q_{+}$.

The main theorem of this section (Theorem 4.2) is based on the following Assumption 4.1, which replaces the convexity assumption made in Theorem 3.1. Remark 4.3 below shows that it is a strengthened version of Scarf's (1986) distributiveness assumption. Condition (i) of Assumption 4.1 means increasing returns to scale. Condition (ii) means that nonmarket commodities are only used as inputs and cannot be produced; in particular it excludes nonmarket intermediate commodities. Condition (iii) says that $c_{I}^{w i c}$ can be continuously extended from $Q_{+} \times \stackrel{\circ}{\mathbf{R}}_{+}$to $Q \times \stackrel{\circ}{\mathbf{R}}_{+}$. Condition (iv) means the convexity of each production possibility set. Condition (v) means diminishing marginal rate of technical substitution. 


\section{ASSUMPTION 4.1 (Strongly Distributive Total Production Set of}

the Grand Coalition)

(i) For any $y_{I} \in Y_{I}$ and any real number $\alpha>1$, it follows that $\alpha y_{I} \in Y_{I}$;

(ii) for any $i \in I, Y_{i} \subset \mathbf{R}^{k_{m}|T|} \times\left(-\mathbf{R}_{+}^{k_{n}|T|}\right)$;

(iii) cost function $c_{S}^{w i c}: Q \times \stackrel{\circ}{+}_{+} \rightarrow \mathbf{R}_{+}$is continuous;

(iv) for any $q \in Q_{+}$, the cost function $g(q, \cdot)$ is strictly quasi-convex;

(v) for each $m$, the input-requirement set $S_{Y_{I}}(m)$ is strictly convex.

A stronger form of exclusion of a nonmarket intermediate commodity is assumed in Theorem 4.2: Condition (1) in the theorem says that if all nonmarket commodities are used as inputs at any type-profile, then a positive profit can be made at any type-profile; in particular, it excludes those divisions that produce only nonmarket intermediate commodities (the firm with such a division will be analyzed in Section 5). Condition (2) says that each division is endowed with all nonmarket commodities at the outset at any type-profile, which again excludes a nonmarket intermediate commodity.

THEOREM 4.2 Let $\mathcal{D}$ be a profit-center game with incomplete information which satisfies Postulates 2.2-2.4 and Assumptions 2.6-2.7 and 4.1. Assume moreover that

(1) for any $n_{i} \in \mathbf{R}^{k_{n}|T|}$ for which $n_{i} \ll 0$, there exists $y_{i}^{\prime} \in Y_{i}$ such that $n_{i} \leq n_{i}^{\prime}, y_{1 i}^{\prime}$ is $\mathcal{T}_{i}$-measurable, and $p \cdot m_{i}^{\prime}(t)>0$ for all $t \in T$; and

(2) $r_{i}\left(t_{i}\right) \gg 0$, for every $i \in I$ and every $t \in T$.

Then there exists a full-information revealing core plan of the game.

REMARK 4.3 Consider a production economy in which the commodities are classified into two categories, say $\kappa_{1}$ products and $\kappa_{2}$ production factors. Scarf (1986) defined a distributive set as a production set $Y\left(\subset \mathbf{R}^{\kappa_{1}} \times\left(-\mathbf{R}_{+}^{\kappa_{2}}\right)\right)$ which has the following property (here, the usual sign convention is adopted, so a coordinate of activity $(y, z) \in Y, y \in \mathbf{R}^{\kappa_{1}}$ and $z \in-\mathbf{R}_{+}^{\kappa_{2}}$, is positive or negative according to whether the corresponding commodity is a net output or a net input):

For any finitely many activities $\left(y^{h}, z^{h}\right) \in Y, h=1, \cdots, l$ and any nonnegative real numbers $\alpha_{h}, h=1, \cdots, l$ such that $\sum_{h=1}^{l} \alpha_{h} z^{h} \leq$ $z^{i}$ for all $i=1, \cdots, l$, it follows that $\sum_{h=1}^{l} \alpha_{h}\left(y^{h}, z^{h}\right) \in Y$. 
Now, given cost function $g(q, \cdot): \mathbf{R}^{\kappa_{1}} \rightarrow \mathbf{R}$, define the "production set" $\bar{Y}$ by

$$
\bar{Y}:=\left\{(m,-w) \in \mathbf{R}^{\kappa_{1}} \times\left(-\mathbf{R}_{+}\right) \mid g(q, m) \leq w\right\} .
$$

Then, it is not difficult to show that if $g(q, \cdot)$ is quasi-convex and subhomogeneous, then $\bar{Y}$ is a distributive set. (The proof is a straightforward application of the support theorem.)

REMARK 4.4 Consider the coalitional total production set approach. Theorem 4.2 still holds true, if $Y(S) \subset Y(I)$ for every $S \in \mathcal{I}$, and if $Y(I)$ satisfies all the conditions that are imposed on $Y$ in Theorem 4.2.

REMARK 4.5 The conditions in Theorem 4.2 assume implicitly that adjustment of non-commodity resources (such as a plant) and/or effort to each net output plan plays an important role. Indeed, the specific instance of $Y_{i}=\prod_{t \in T} Y_{i}(t)$ is in general inconsistent with conditions (i) and (iv) of Assumption 4.1. To see this, consider a simple example of: $|I|=1, T=\left\{t, t^{\prime}\right\}$, $Y(t)=Y\left(t^{\prime}\right),\left|K_{n}\right|=\left|K_{1 n}\right|=1,\left|K_{m}\right|=1, p=1$ and $q(t)=q\left(t^{\prime}\right)=1 / 2$. Choose any

$$
(y(t), \mathbf{0}),\left(\mathbf{0}, y^{\prime}\left(t^{\prime}\right)\right) \in Y,
$$

so that

$$
q(t) n(t)=q\left(t^{\prime}\right) n^{\prime}\left(t^{\prime}\right)=g(q, m)=g\left(q, m^{\prime}\right) .
$$

Then, by strict quasi-convexity of $g(q, \cdot)$,

$$
\left(\frac{1}{2} y(t), \frac{1}{2} y^{\prime}\left(t^{\prime}\right)\right) \in \text { interior of } Y(t) \times Y\left(t^{\prime}\right),
$$

which contradicts condition (i).

REMARK 4.6 Let $\left(x_{S}, y\right)$ be an optimal solution to Problem $P_{S}(q, \eta)$. To prove that it is weakly Bayesian incentive-compatible, it suffices to show

$$
\forall i \in S: \forall t_{i}, t_{i}^{\prime} \in T_{i}: E\left(x_{i} \mid t_{i}\right)=E\left(x_{i} \mid t_{i}^{\prime}\right),
$$

in view of Fact 6.2 (ii). Suppose

$$
\exists \bar{t}_{i}, \overline{\bar{t}}_{i} \in T_{i}: E\left(x_{i} \mid \bar{t}_{i}\right)<E\left(x_{i} \mid \overline{\bar{t}}_{i}\right) .
$$


Then, by subtracting a positive constant from $x_{i}$ on the minimal member of $\mathcal{T}_{i}$ that contains $\left\{\overline{\bar{t}}_{i}\right\} \times T_{I \backslash\{i\}}$, one obtains a $\mathcal{T}_{S}$-measurable function $x_{i}^{\prime}\left(<x_{i}\right)$ for which

$$
\eta \leq \sum_{i \in S} \min _{t \in T} E\left(x_{i}^{\prime} \mid \mathcal{T}_{i}\right)(t) .
$$

Then, there exists $y_{i}^{\prime} \in Y_{i}$ such that

$$
\begin{gathered}
\forall t \in T: \sum_{i \in S} x_{i}^{\prime} \leq \sum_{i \in S} p \cdot m_{i}^{\prime}(t), \text { and } \\
-\sum_{t \in T} q(t) \cdot n^{\prime}(t)<-\sum_{t \in T} q(t) \cdot n(t),
\end{gathered}
$$

which contradicts the optimality of $\left(x_{S}, y\right)$.

\section{Complementary Suppliers of Nonmarket Intermediate Commodities}

The preceding two sections focused on the role of returns to scale, nonincreasing or increasing. The present section, on the other hand, focuses on the role of a structural relationship between two types of divisions, the suppliers and the customers. The suppliers produce and supply to the customers nonmarket intermediate commodities. The customers use these nonmarket intermediate commodities, produce market commodities and bring in profit to the firm. The products of all suppliers are needed for each customer's production activities, hence the term complementary suppliers. No assumption on returns to scale is made. The idea about this relationship goes back to Radner (1992, Subsection 7.2). The following are a formal treatment of its simplified version in the context of incomplete information.

The division set, $I$, is partitioned into the supplier set, $I_{s}$, and the customer set, $I_{c}$. Let $K_{n}$, be the set of nonmarket intermediate commodities, a subset of $K_{n}$. A net output plan $y_{i}$ is denoted by $\left(m_{i}, n_{s i}, n_{z i}\right)$, where the subvectors $n_{s i}$ and $n_{z i}$ correspond to $K_{n_{s}}$ and $K_{n} \backslash K_{n_{s}}$, respectively.

The following Assumption 5.1 highlights the role of complementary suppliers of nonmarket intermediate commodities. In particular, (i) says that a supplier can produce only nonmarket intermediate commodities; (ii) says that all the suppliers are needed in order to produce all nonmarket intermediate 
commodities; (iii) says that for a customer to produce a market commodity, all the nonmarket intermediate commodities are needed; (iv) says that a coalition of a customer and all the suppliers can make a positive profit if all the nonmarket non-intermediate commodity resources are available (even if in arbitrarily small amount); (v)(a) says that no nomarket intermediate commodities are available as initial resources (so that they have to be produced by suppliers); and (v)(b) says that all the nonmarket non-intermediate commodities are available as initial resources in the grand coalition.

ASSUMPTION 5.1 ([Complementary Supplier]-Customer Relationship)

(i) For each supplier $i \in I_{s}$, if $y_{i} \in Y_{i}$, then $m_{i} \leq 0$ and $n_{z i} \leq 0$.

(ii) If $y_{i} \in Y_{i}$ for each supplier $i_{s}$ and if $\sum_{i \in I_{s}} n_{s i}(t) \gg 0$ for some $t \in T$, then for this $t, n_{s i}(t)>0$ for every $i \in I_{s}$.

(iii) For each customer $i \in I_{c}$ and each $t \in T$, if $\left[y_{i} \in Y_{i}\right.$ and $\left.\neg n_{s i}(t) \ll 0\right]$, then $m_{i}(t) \leq 0$.

(iv) For any $i \in I_{c}$ and any $\varepsilon>0$, there exists $y_{I_{s} \cup\{i\}} \in Y_{I_{s} \cup\{i\}}$ such that each $y_{1 j}$ is $\mathcal{T}_{j}$-measurable, $j \in I_{s} \cup\{i\}$, and

$$
\begin{array}{ll}
\forall(t, a) \in T \times K_{n_{c}}: & \sum_{j \in I_{s} \cup\{i\}} n_{z j}(t, a)>-\varepsilon, \\
\forall t \in T: & p \cdot \sum_{j \in I_{s} \cup\{i\}} m_{j}(t)>0 .
\end{array}
$$

(v)(a) For each division $i \in I, r_{s i}=\mathbf{0}\left(\in \mathbf{R}^{k_{n_{s}}|T|}\right)$;

(b) $\sum_{i \in I} r_{z i} \gg 0$.

A customer has to use some nonmarket non-intermediate commodities in the first interim period, e.g., human asset. Therefore, Assumption 2.7 is consistent with the present setup of supplier-customer relationship. The following existence theorem is valid regardless of the nature of returns to scale.

THEOREM 5.2 Let $\mathcal{D}$ be a profit-center game with incomplete information which satisfies Postulates 2.2-2.4 and Assumptions 2.6-2.7 and 5.1. Then there exists a full-information revealing core plan of the game.

REMARK 5.3 Theorem 5.2 still holds true in the specific instance of a production set given by $Y_{i}=\prod_{t \in T} Y_{i}(t)$. 


\section{Some Details and Proofs}

This section begins with the precise definition of Bayesian incentive compatibility and its several characterization results (Facts 6.1 and 6.2).

A pretension function of division $i$ is a function $\sigma: T_{i} \rightarrow T_{i}$, which says that when its true type is $t_{i}$, it acts (makes a choice) as though its type were $\sigma\left(t_{i}\right)$. Given any algebra $\mathcal{B}_{i}$ on $T_{i}$, denote by endo $\left(T_{i}, \mathcal{B}_{i}\right)$ the set of all functions that map each $t_{i}$ into the minimal set of $\mathcal{B}_{i}$ that contains $t_{i}$. Indeed, let $\mathcal{P}$ be the partition of $T_{i}$ that consists of the minimal nonempty members of $\mathcal{B}_{i}$. Then,

$$
\text { endo }\left(T_{i}, \mathcal{B}_{i}\right):=\left\{\sigma: T_{i} \rightarrow T_{i} \mid \forall P \in \mathcal{P}: \sigma(P) \subset P\right\} \text {. }
$$

If every member of coalition $S$ has information structure $\mathcal{B}_{i}$ about division $i$ 's type, then $i$ can only choose a pretension function $\sigma \in$ endo $\left(T_{i}, \mathcal{B}_{i}\right)$.

Suppose the members of $S$ are deliberating on whether or not to agree on a plan $\left(x_{S}, y_{S}\right)$. In the first period, division $i$ 's information structure is given as $\mathcal{T}_{i}$, which is private. So, division $i$ is not caught in the first period no matter which choice it makes from $\left\{y_{1 i}\left(t_{i}\right) \mid t_{i} \in T_{i}\right\}$; that is, it can make a choice according to any pretension function $\sigma \in$ endo $\left(T_{i},\left\{\emptyset, T_{i}\right\}\right)$, so that when division $i$ 's true type is $\bar{t}_{i}$, it makes the choice $y_{1 i}\left(\sigma\left(\bar{t}_{i}\right)\right)$. By acting according to the function $y_{1 i} \circ \sigma$, division $i$ having its true type $\bar{t}_{i}$ passes on to all the other members of $S$ the information that event $A:=y_{1 i}^{-1}\left(y_{1 i} \circ \sigma\left(\bar{t}_{i}\right)\right)$ has occurred. This information may be false, that is, $\bar{t}_{i}$ may not be a member of $A$, but the other members take it as $i$ 's testimony about itself and expect that $i$ will act according to this information in the second period, that is, $i$ will have to make a choice from $\left(x_{i}, y_{2 i}\right)(A)$ in the second period. Therefore, $i$ 's pretension function in the second period has to be of the form $\tau \circ \sigma$ for some $\tau \in$ endo $\left(T_{i}, \mathcal{A}\left(y_{1 i}\right)\right)$.

The present concept of Bayesian incentive compatibility says that division $i$ cannot benefit from any pair of pretension functions that are not caught. Formally, a plan $\left(x_{S}, y_{S}\right)$ is called Bayesian incentive-compatible, if for all $i \in S$, all $\sigma \in$ endo $\left(T_{i},\left\{\emptyset, T_{i}\right\}\right)$, and all $\tau \in$ endo $\left(T_{i}, \mathcal{A}\left(y_{1 i}\right)\right)$,

$$
\forall t \in T: E\left(x_{i} \mid \hat{\mathcal{T}}_{i}\left(y_{1 S}\right)\right)(t) \geq E\left(x_{i} \circ(\tau \circ \sigma, \mathrm{id}) \mid \hat{\mathcal{T}}_{i}\left(y_{1 S}\right)\right)(t)
$$

where id is the identity map on $T_{S \backslash\{i\}}$.

The first characterization (Fact 6.1) is proved in the same way as in the first paragraph of Ichiishi, Idzik and Zhao (1994, Section 4, p. 159). 
FACT 6.1 Plan $\left(x_{S}, y_{S}\right) \in F_{S}^{\prime}$ is Bayesian incentive-compatible, iff for all $i \in S$, all $\sigma \in$ endo $\left(T_{i},\left\{\emptyset, T_{i}\right\}\right)$, and all $t \in T, E\left(x_{i} \mid \hat{\mathcal{T}}_{i}\left(y_{1 S}\right)\right)(t) \geq$ $E\left(x_{i} \circ(\sigma, \mathrm{id}) \mid \hat{\mathcal{T}}_{i}\left(y_{1 s}\right)\right)(t)$.

FACT 6.2 (i) Plan $\left(x_{S}, y_{S}\right) \in F_{S}^{\prime}$ is Bayesian incentive-compatible, iff

$$
\begin{aligned}
& \forall i \in S: \forall t_{i}, t_{i}^{\prime} \in T_{i}: \forall \bar{t}_{I \backslash\{i\}} \in T_{I \backslash\{i\}}: \\
& \quad E\left(x_{i} \mid \hat{\mathcal{T}}_{i}\left(y_{1 S}\right)\right)\left(t_{i}, \bar{t}_{I \backslash\{i\}}\right)=E\left(x_{i} \mid \hat{\mathcal{T}}_{i}\left(y_{1 S}\right)\right)\left(t_{i}^{\prime}, \bar{t}_{I \backslash\{i\}}\right),
\end{aligned}
$$

(ii) For any weakly Bayesian incentive-compatible plan $\left(x_{S}, y_{S}\right)$ and for any $i \in S, E\left(x_{i} \mid \mathcal{T}_{i}\right)$ is a constant function on $T$.

Proof (i) The sufficiency is trivial, so it suffices to show the necessity. Choose any $\sigma \in$ endo $\left(T_{i},\left\{\emptyset, T_{i}\right\}\right)$ and any $\bar{t} \in T$. Let $\left\{\bar{t}_{i}\right\} \times A(\subset T)$ be the minimal element of $\hat{T}_{i}\left(y_{1 S}\right)$ that contains $\bar{t}$. Then, by the present setup of $\pi(t)=\prod_{i} \pi_{i}\left(t_{i}\right)$,

$$
\begin{aligned}
E & \left(x_{i} \circ(\sigma, \mathrm{id}) \mid \hat{\mathcal{T}}_{i}\left(y_{1 S}\right)\right)(\bar{t}) \\
& =\frac{1}{\prod_{j \in S \backslash\{i\}} \pi_{j}\left(A_{j}\right)} \sum_{t_{S \backslash\{i\}} \in A_{S}}\left(\prod_{j \neq i} \pi_{j}\left(t_{j}\right)\right) x_{i}\left(\sigma\left(\bar{t}_{i}\right), t_{S \backslash\{i\}}\right) \\
& =E\left(x_{i} \mid \hat{\mathcal{T}}_{i}\left(y_{1 S}\right)\right)\left(\sigma\left(\bar{t}_{i}\right), \bar{t}_{I \backslash\{i\}}\right)
\end{aligned}
$$

(here, set $A$, which is of the form $\prod_{j \in S \backslash\{i\}} A_{j} \times \prod_{j \in I \backslash S} T_{j}$, is identified with $\prod_{j \in S \backslash\{i\}} A_{j}$ ). By Fact 6.1 , the Bayesian incentive compatibility says: for all $\sigma \in$ endo $\left(T_{i},\left\{\emptyset, T_{i}\right\}\right)$,

$$
E\left(x_{i} \mid \hat{\mathcal{T}}_{i}\left(y_{1 S}\right)\right)(\bar{t}) \geq E\left(x_{i} \circ(\sigma, \mathrm{id}) \mid \hat{\mathcal{T}}_{i}\left(y_{1 S}\right)\right)(\bar{t}) .
$$

By (1) and (2),

$$
E\left(x_{i} \mid \hat{\mathcal{T}}_{i}\left(y_{1 S}\right)\right)(\bar{t}) \geq E\left(x_{i} \mid \hat{\mathcal{T}}_{i}\left(y_{1 S}\right)\right)\left(\sigma\left(\bar{t}_{i}\right), \bar{t}_{I \backslash\{i\}}\right) .
$$

This is true for any $\bar{t}_{i}$, any $\sigma$, and any $\bar{t}_{\Gamma \backslash\{i\}}$. So,

$$
E\left(x_{i} \mid \hat{\mathcal{T}}_{i}\left(y_{1 S}\right)\right)(\bar{t})=E\left(x_{i} \mid \hat{\mathcal{T}}_{i}\left(y_{1 S}\right)\right)\left(\sigma\left(\bar{t}_{i}\right), \bar{t}_{I \backslash\{i\}}\right),
$$


which is the required result.

(ii) Choose any $\sigma \in$ endo $\left(T_{i},\left\{\emptyset, T_{i}\right\}\right)$. By the present setup of $\pi(t)=$ $\prod_{i} \pi_{i}\left(t_{i}\right)$

$$
\begin{aligned}
& E\left(x_{i} \circ(\sigma, \mathrm{id}) \mid \bar{t}_{i}\right)=\sum_{t_{S \backslash\{i\}} \in T_{S \backslash\{i\}}}\left(\prod_{j \neq i} \pi_{j}\left(t_{j}\right)\right) x_{i}\left(\sigma\left(\bar{t}_{i}\right), t_{S \backslash\{i\}}\right) \\
& =E\left(x_{i} \mid \sigma\left(\bar{t}_{i}\right)\right) \text {. }
\end{aligned}
$$

Then the weak Bayesian incentive compatibility says

$$
\forall \sigma \in \text { endo }\left(T_{i},\left\{\emptyset, T_{i}\right\}\right): \forall t_{i} \in T_{i}: E\left(x_{i} \mid t_{i}\right) \geq E\left(x_{i} \mid \sigma\left(t_{i}\right)\right),
$$

hence the required result.

Recall that $r_{1 i}$ is a function only of $t_{i} \in T_{i}$, and so is variable $n_{1 i}$, the subvector of $n_{i}$ that corresponds to $K_{1 n}$ (Postulate 2.2). For a net output plan $y_{i}: T \rightarrow \mathbf{R}^{k}$, denote by $y_{i}(t, a)$ the $a$ th coordinate of $y_{i}(t),(t, a) \in T \times K$.

\section{LEMMA 6.3 Suppose}

$$
\forall t \in T:-\sum_{i \in I} n_{1 i}\left(t_{i}\right) \leq \sum_{i \in I} r_{1 i}\left(t_{i}\right) .
$$

Then, for each $i$ there exists a function $\overline{\bar{n}}_{1 i}: T_{i} \rightarrow \mathbf{R}^{k_{1 n}}$ such that $\overline{\bar{n}}_{1 i} \leq n_{1 i}$ and

$$
\forall t \in T:-\sum_{i \in I} \overline{\bar{n}}_{1 i}\left(t_{i}\right)=\sum_{i \in I} r_{1 i}\left(t_{i}\right) \text {. }
$$

Proof Step 1. It will be shown first that if

$$
\exists \bar{t} \in T: \quad \exists \hat{a} \in K_{1 n}:-\sum_{i \in I} n_{i}\left(\bar{t}_{i}, \hat{a}\right)<\sum_{i \in I} r_{i}\left(\bar{t}_{i}, \hat{a}\right),
$$

then

$$
\exists i: \forall t_{I \backslash\{i\}}:-n_{i}\left(\bar{t}_{i}, \hat{a}\right)-\sum_{j \in I \backslash\{i\}} n_{j}\left(t_{j}, \hat{a}\right)<r_{i}\left(\bar{t}_{i}, \hat{a}\right)+\sum_{j \in I \backslash\{i\}} r_{j}\left(t_{j}, \hat{a}\right) .
$$

This will be done in the following Steps 2-4. Only in these Steps 2-4, simplifying notation will be used, so that $n_{i}\left(t_{i}, \hat{a}\right), n_{i}\left(\bar{t}_{i}, \hat{a}\right), n_{i}\left(t_{i}^{\prime}, \hat{a}\right), r_{i}\left(t_{i}, \hat{a}\right), r_{i}\left(\bar{t}_{i}, \hat{a}\right)$, 
$r_{i}\left(t_{i}^{\prime}, \hat{a}\right)$, etc., will be denoted by $n_{i}, \bar{n}_{i}, n_{i}^{\prime}, r_{i}, \bar{r}_{i}, r_{i}^{\prime}$, etc., respectively, and the set divisions $I$ will be identified with the set of integers $\{1,2, \cdots, I\}$ (where the last integer in the set is $|I|$, by abuse of notation). Condition (4) is then re-written as:

$$
\exists \bar{t}:-\bar{n}_{1}-\bar{n}_{2}-\bar{n}_{3}-\cdots-\bar{n}_{I}<\bar{r}_{1}+\bar{r}_{2}+\bar{r}_{3}+\cdots+\bar{r}_{I} .
$$

Step 2. If for all $\left(t_{2}, t_{3}, \cdots, t_{I}\right)$,

$$
-\bar{n}_{1}-n_{2}-n_{3}-\cdots-n_{I}<\bar{r}_{1}+r_{2}+r_{3}+\cdots+r_{I},
$$

then there is nothing to prove. So assume

$$
\exists\left(t_{2}^{\prime}, t_{3}^{\prime}, \cdots, t_{I}^{\prime}\right):-\bar{n}_{1}-n_{2}^{\prime}-n_{3}^{\prime}-\cdots-n_{I}^{\prime}=\bar{r}_{1}+r_{2}^{\prime}+r_{3}^{\prime}+\cdots+r_{I}^{\prime} .
$$

Then,

$$
\forall t_{1}:-n_{1}-\bar{n}_{2}-\bar{n}_{3}-\cdots-\bar{n}_{I}<r_{1}+\bar{r}_{2}+\bar{r}_{3}+\cdots+\bar{r}_{I} .
$$

Indeed, if there exists $\tilde{t}_{1}$ for which

$$
-\tilde{n}_{1}-\bar{n}_{2}-\bar{n}_{3}-\cdots-\bar{n}_{I}=\tilde{r}_{1}+\bar{r}_{2}+\bar{r}_{3}+\cdots+\bar{r}_{I},
$$

then by adding the two equalities,

$$
\begin{aligned}
& \left(-\bar{n}_{1}-\bar{n}_{2}-\bar{n}_{3}-\cdots-\bar{n}_{I}\right)+\left(-\tilde{n}_{1}-n_{2}^{\prime}-n_{3}^{\prime}-\cdots-n_{I}^{\prime}\right) \\
= & \left(-\bar{n}_{1}-n_{2}^{\prime}-n_{3}^{\prime}-\cdots-n_{I}^{\prime}\right)+\left(-\tilde{n}_{1}-\bar{n}_{2}-\bar{n}_{3}-\cdots-\bar{n}_{I}\right) \\
= & \left(\bar{r}_{1}+r_{2}^{\prime}+r_{3}^{\prime}+\cdots+r_{I}^{\prime}\right)+\left(\tilde{r}_{1}+\bar{r}_{2}+\bar{r}_{3}+\cdots+\bar{r}_{I}\right) \\
= & \left(\bar{r}_{1}+\bar{r}_{2}+\bar{r}_{3}+\cdots+\bar{r}_{I}\right)+\left(\tilde{r}_{1}+r_{2}^{\prime}+r_{3}^{\prime}+\cdots+r_{I}^{\prime}\right) .
\end{aligned}
$$

By (4),

$$
-\tilde{n}_{1}-n_{2}^{\prime}-n_{3}^{\prime}-\cdots-n_{I}^{\prime}>\tilde{r}_{1}+r_{2}^{\prime}+r_{3}^{\prime}+\cdots+r_{I}^{\prime},
$$

which contradicts $(3)$.

Step 3. If for all $\left(t_{1}, t_{3}, t_{4}, \cdots, t_{I}\right)$,

$$
-n_{1}-\bar{n}_{2}-n_{3}-\cdots-n_{I}<r_{1}+\bar{r}_{2}+r_{3}+\cdots+r_{I},
$$

then there is nothing to prove. So assume

$$
\exists\left(t_{1}^{\prime}, t_{3}^{\prime}, t_{4}^{\prime}, \cdots, t_{I}^{\prime}\right):-n_{1}^{\prime}-\bar{n}_{2}-n_{3}^{\prime}-\cdots-n_{I}^{\prime}=r_{1}^{\prime}+\bar{r}_{2}+r_{3}^{\prime}+\cdots+r_{I}^{\prime} .
$$


Then,

$$
\forall\left(t_{1}, t_{2}\right):-n_{1}-n_{2}-\bar{n}_{3}-\cdots-\bar{n}_{I}<r_{1}+r_{2}+\bar{r}_{3}+\cdots+\bar{r}_{I} .
$$

Indeed, if there exists $\left(\tilde{t}_{1}, \tilde{t}_{2}\right)$ for which

$$
-\tilde{n}_{1}-\tilde{n}_{2}-\bar{n}_{3}-\cdots-\bar{n}_{I}=\tilde{r}_{1}+\tilde{r}_{2}+\bar{r}_{3}+\cdots+\bar{r}_{I},
$$

then by adding the two equalities,

$$
\begin{aligned}
& \left(-n_{1}^{\prime}-\bar{n}_{2}-\bar{n}_{3}-\cdots-\bar{n}_{I}\right)+\left(-\tilde{n}_{1}-\tilde{n}_{2}-n_{3}^{\prime}-\cdots-n_{I}^{\prime}\right) \\
= & \left(r_{1}^{\prime}+\bar{r}_{2}+\bar{r}_{3}+\cdots+\bar{r}_{I}\right)+\left(\tilde{r}_{1}+\tilde{r}_{2}+r_{3}^{\prime}+\cdots+r_{I}^{\prime}\right) .
\end{aligned}
$$

By (5),

$$
-\tilde{n}_{1}-\tilde{n}_{2}-n_{3}^{\prime}-\cdots-n_{I}^{\prime}>\tilde{r}_{1}+\tilde{r}_{2}+r_{3}^{\prime}+\cdots+r_{I}^{\prime},
$$

which contradicts (3).

Step 4. Continuing this way, one can prove that if for each $i, 1 \leq i \leq I-1$, there exists $\left(t_{1}^{\prime}, \cdots, t_{i-1}^{\prime}, t_{i+1}^{\prime}, \cdots, t_{I}^{\prime}\right)$ for which

$$
\begin{aligned}
& -n_{1}^{\prime}-n_{2}^{\prime}-\cdots-n_{i-1}^{\prime}-\bar{n}_{i}-n_{i+1}^{\prime}-n_{i+2}^{\prime}-\cdots-n_{I}^{\prime} \\
= & r_{1}^{\prime}+r_{2}^{\prime}+\cdots+r_{i-1}^{\prime}+\bar{r}_{i}+r_{i+1}^{\prime}+r_{i+2}^{\prime}+\cdots+r_{I}^{\prime},
\end{aligned}
$$

then for all $\left(t_{1}, t_{2}, \cdots, t_{I-1}\right)$,

$$
-n_{1}-n_{2}-\cdots-n_{I-1}-\bar{n}_{I}<r_{1}+r_{2}+\cdots+r_{I-1}+\bar{r}_{I} .
$$

This is precisely the required result stated in Step 1.

Step 5. Suppose (4) holds true. Then, by Step 1, one may choose $i_{1}$ and $\varepsilon>0$ such that for all $t_{I \backslash\left\{i_{1}\right\}}$

$$
-\left[n_{i_{1}}\left(\bar{t}_{i_{1}}, \hat{a}\right)-\varepsilon\right]-\sum_{j \in I \backslash\left\{i_{1}\right\}} n_{j}\left(t_{j}, \hat{a}\right) \leq r_{i_{1}}\left(\bar{t}_{i_{1}}, \hat{a}\right)+\sum_{j \in I \backslash\left\{i_{1}\right\}} r_{j}\left(t_{j}, \hat{a}\right),
$$

with equality for at least one $t_{\left.\Upsilon \backslash i_{1}\right\}}$. Define $n_{1 i}^{(1)}: T_{i} \rightarrow \mathbf{R}^{k_{1 n}}$ by

$$
n_{i}^{(1)}\left(t_{i}, a\right):= \begin{cases}n_{i_{1}}\left(\bar{t}_{i_{1}}, \hat{a}\right)-\varepsilon, & \text { if }\left(i, t_{i}, a\right)=\left(i_{1}, \bar{t}_{i_{1}}, \hat{a}\right) \\ n_{i}\left(t_{i}, a\right), & \text { otherwise. }\end{cases}
$$

The function $n_{1 I}^{(1)}$ satisfies (3), and $n_{1 i}^{(1)} \leq n_{1 i}$. Moreover, by the choice of $\varepsilon$, the number of strict inequalities in (3) is less for $n_{1 I}^{(1)}$ than for $n_{1 I}$. If (4) 
is true for $n_{1 I}^{(1)}$, repeat the procedure (Steps 1-4) and obtain $n_{1 I}^{(2)}\left(<n_{1 I}^{(1)}\right)$. At each repetition, the number of strict inequalities in (3) strictly decreases. After finitely many steps, one obtains equality in (3).

Sets $\hat{F}_{S}, S \in \mathcal{I}$, are neither convex nor closed, which causes difficulty in proving existence theorems (Theorem 3.1 and 4.2). To overcome this difficulty, Radner's (1979) technique is adopted: Instead of using sets $F_{S}^{\prime}$, one considers a larger set of $\left(x_{S}, y_{S}\right)$ such that $\left(x_{S}, y_{2 S}\right)$ is $\mathcal{T}_{S}$-measurable and each $y_{1 i}$ is $\mathcal{T}_{i}$-measurable. A plan $\left(x_{I}^{*}, y_{I}^{*}\right)$ which satisfies all the equilibrium conditions (Definition 2.5) except the information-pooling rule (Postulate $2.3)$ is obtained. In fact, one looks for $\left(x_{I}^{*}, y_{I}^{*}\right)$ such that each $n_{1 i}^{*}$ is 1-1. Given the injectiveness of $n_{1 i}^{*}, i \in I$, the algebra generated by $y_{1}^{*}$ is the finest algebra $2^{T}$, so $\left(x_{I}^{*}, y_{I}^{*}\right)$ satisfies the information-pooling rule.

\section{Proof of Theorem 3.1 Define}

$$
\begin{aligned}
G_{S} & :=\left\{\left(x_{S}, y_{S}\right) \in F_{S} \mid \forall i \in S: y_{1 i} \text { is } \mathcal{T}_{i} \text {-measurable }\right\}, \\
G_{S}^{w i c} & :=\left\{\begin{array}{l|l}
\left(x_{S}, y_{S}\right) \in G_{S} & \begin{array}{l}
\left(x_{S}, y_{S}\right) \text { is weakly } \\
\text { Bayesian incentive-compatible }
\end{array}
\end{array}\right\},
\end{aligned}
$$

and define the non-side-payment games, $\hat{V}: \mathcal{I} \rightarrow \mathbf{R}^{I}$ and $V^{\text {wic }}: \mathcal{I} \rightarrow \mathbf{R}^{I}$, by

$$
\begin{aligned}
\hat{V}(S) & :=\left\{u_{I} \in \mathbf{R}^{I} \mid \exists\left(x_{S}, y_{S}\right) \in \hat{F}_{S}: \forall i \in S: u_{i} \leq E x_{i}\right\}, \\
V^{w i c}(S) & :=\left\{u_{I} \in \mathbf{R}^{I} \mid \exists\left(x_{S}, y_{S}\right) \in G_{S}^{\text {wic }}: \forall i \in S: u_{i} \leq E x_{i}\right\},
\end{aligned}
$$

where $E x_{i}:=\sum_{t \in T} x_{i}(t) \pi(t)$, the $e x$ ante expected profit attributed to $i$.

Game $V^{\text {wic }}$ is balanced. Indeed, let $\mathcal{B}$ be a balanced subfamily of $\mathcal{I}$ with the associated balancing coefficients $\left\{\lambda_{S}\right\}_{S \in \mathcal{B}}$, and choose any $u \in$ $\cap_{S \in \mathcal{B}} V^{\text {wic }}(S)$. For each $S \in \mathcal{B}$, there exists $\left(x_{i}^{(S)}, y_{i}^{(S)}\right)_{i \in S} \in G_{S}^{\text {wic }}$ such that $u_{i} \leq E x_{i}^{(S)}$ for every $i \in S$. Define $\left(x_{I}, y_{I}\right)$ by

$$
\left(x_{i}, y_{i}\right):=\sum_{S \in \mathcal{B}: S \ni i} \lambda_{S}\left(x_{i}^{(S)}, y_{i}^{(S)}\right) .
$$

By the present convexity condition on $Y_{i},\left(x_{I}, y_{I}\right) \in G_{I}$. Since each $E\left(x_{i}^{(\mathcal{S})}\right.$ $\left.\mathcal{T}_{i}\right)$ is a constant function on $T$ by Fact 6.2 (ii), so is $E\left(x_{i} \mid \mathcal{T}_{i}\right)$ as a weighted average of these functions. Therefore, $\left(x_{I}, y_{I}\right) \in G_{I}^{w i c}$. Since $u_{i} \leq E x_{i}$, it follows that $u \in V^{\text {wic }}$. 
By the Scarf theorem (see, e.g., Scarf (1973, Theorem 8.3.6, p. 211)), one can choose $u^{*}$ in the core of game $V^{\text {wic }}$. Let $\left(\bar{x}_{I}, \bar{y}_{I}\right) \in G_{I}^{\text {wic }}$ be the plan that gives rise to $u^{*}$. By Fact $6.1, G_{S}^{w i c} \supset F_{S}^{\prime \prime}$, so $u^{*}$ cannot be improved upon by any proper coalitions in game $V$.

By Lemma 6.3, for each $i$ there exists a $\mathcal{T}_{i}$-measurable function $\overline{\bar{n}}_{1 i}: T \rightarrow$ $\mathbf{R}^{k_{1 n}}$ such that

$$
\overline{\bar{n}}_{1 i} \leq \bar{n}_{1 i}
$$

and

$$
-\sum_{i \in I} \overline{\bar{n}}_{1 i}=\sum_{i \in I} r_{1 i} .
$$

Each $\overline{\bar{n}}_{1 i}$ is 1-1 on $T_{i}$. Indeed, this follows from the identity,

$$
\forall t \in T: \overline{\bar{n}}_{1 i}\left(t_{i}\right)+r_{1 i}\left(t_{i}\right)=-\sum_{j \in I \backslash\{i\}}\left(\overline{\bar{n}}_{1 j}\left(t_{j}\right)+r_{1 j}\left(t_{j}\right)\right),
$$

and the facts that the right-hand side is constant once $t_{I \backslash\{i\}}$ is fixed, and that $r_{1 i}(\cdot)$ is $1-1$ on $T_{i}$ (Assumption 2.7 (ii)).

Define $\overline{\bar{x}}_{i}:=E\left(\bar{x}_{i} \mid \mathcal{T}_{i}\right)$, and define the plan $\left(x_{I}^{*}, y_{I}^{*}\right)$ by

$$
x_{I}^{*}:=\overline{\bar{x}}_{I}, m_{1 I}^{*}:=\bar{m}_{1 I}, n_{1 I}^{*}:=\overline{\bar{n}}_{1 I}, y_{2 I}^{*}:=\bar{y}_{2 I} .
$$

By the free disposal assumption (Assumption 2.6 (iii)), $\left(\bar{x}_{I}, y_{I}^{*}\right) \in G_{I}^{\text {wic }}$. Since $n_{1 i}^{*}$ is $1-1$ on $T_{i}$ for all $i$, it follows that $\hat{\mathcal{T}}_{i}\left(y_{1 I}^{*}\right)$ is the finest algebra $2^{T}$ for every $i$, so $\left(\bar{x}_{I}, y_{I}^{*}\right) \in F_{I}^{\prime}$. By the headquarters' insurability (Postulate 2.4), $\left(x_{I}^{*}, y_{I}^{*}\right) \in \hat{F}_{I}$. Notice that $E \overline{\bar{x}}_{i}=E \bar{x}_{i} \geq u_{i}^{*}$. It is also easy to check that $u^{*}$ lies in the Pareto frontier of $\hat{V}(I)$. Thus, $u^{*}$ is in the core of game $\hat{V}$. The plan $\left(x_{I}^{*}, y_{I}^{*}\right)$ is full-information revealing.

The plan $\left(x_{I}^{*}, y_{I}^{*}\right)$ is the required core plan of the profit-center game. Indeed, if there exist $S \in \mathcal{I}$ and $\left(x_{S}, y_{S}\right) \in \hat{F}_{S}$ such that for all $i \in S$,

$$
\begin{array}{ll}
\forall t \in T: & E\left(x_{i} \mid \hat{\mathcal{T}}_{i}\left(n_{S}\right)\right)(t) \geq E\left(x_{i}^{*} \mid \hat{\mathcal{T}}_{i}\left(n_{I}^{*}\right)\right)(t), \quad \text { and } \\
\exists t \in T: \quad E\left(x_{i} \mid \hat{\mathcal{T}}_{i}\left(n_{S}\right)\right)(t)>E\left(x_{i}^{*} \mid \hat{\mathcal{T}}_{i}\left(n_{I}^{*}\right)\right)(t),
\end{array}
$$

then $E x_{i}>E x_{i}^{*}$ for every $i \in S$, which contradicts the fact that $u^{*}$ is in the core of game $\hat{V}$.

To prove Theorem 4.2, two results in the earlier literature are recalled: First, consider a static coalition production economy with one primary good 
and one final good, $\left(\left\{\Sigma_{i}, u_{i}, r_{i}\right\}_{i \in I}, c\right)$, where $I$ is a finite set of economic agents, $\Sigma_{i}\left(\subset \mathrm{R}_{+}^{2}\right)$ is a strategy set of agent $i\left(\sigma_{i}:=\left(\eta_{i}, \zeta_{i}\right) \in \Sigma_{i}\right.$ means $i$ 's consumption of $\eta_{i}$ units of the final good and $\zeta_{i}$ units of the primary good), $u_{i}: \Sigma_{i} \rightarrow \mathbf{R}$ is a utility function of agent $i, r_{i}>0$ is the initial resource of primary good held by agent $i$, and $c: \mathbf{R}_{+} \rightarrow \mathbf{R}_{+}$is the cost function, which is common to all coalitions $(c(\eta)$ is the minimal amount of the primary good that is needed to produce $\eta$ units of the final good). The following Theorem 6.4 is due to Scarf (1973):

THEOREM 6.4 (Scarf) Let $\left(\left\{\Sigma_{i}, u_{i}, r_{i}\right\}_{i \in I}, c\right)$ be a coalition production economy with one primary good and one final good. Define the non-sidepayment game $V: \mathcal{I} \rightarrow \mathbf{R}^{I}$ by

$$
V(S):=\left\{\begin{array}{l|l}
u \in \mathbf{R}^{I} & \begin{array}{l}
\exists\left(\eta_{i}, \zeta_{i}\right)_{i \in S} \in \prod_{i \in S} \Sigma_{i}: \\
c\left(\sum_{i \in S} \eta_{i}\right)+\sum_{i \in S} \zeta_{i} \leq \sum_{i \in S} r_{i} \\
\forall i \in S: u_{i} \leq u_{i}\left(\eta_{i}, \zeta_{i}\right)
\end{array}
\end{array}\right\} .
$$

If each $u_{i}$ is nondecreasing in $\mathbf{R}_{+}^{2}$, and if

$$
0<\eta<\eta^{\prime} \Longrightarrow \frac{c(\eta)}{\eta} \geq \frac{c\left(\eta^{\prime}\right)}{\eta^{\prime}},
$$

then the game $V$ is balanced.

The second result is on a family of parameterized static societies: Let $I$ be a finite set of players, let $\Sigma_{i}$ be a strategy space of player $i \in I$, and let $Q$ be a parameter space. Define $\Sigma_{S}:=\prod_{i \in S} \Sigma_{i}$, and set $\Sigma:=\Sigma_{I}$. For each coalition $S \in \mathcal{I}$, a feasible-strategy correspondence $\Phi_{S}: Q \rightarrow \Sigma_{S}$ is given. Correspondence $\Psi: Q \times \Sigma \rightarrow Q$ describes nature's response set $\Psi\left(q, \sigma_{I}\right)(\subset Q)$ to each pair $\left(q, \sigma_{I}\right)$ of a parameter $q$ and a strategy bundle $\sigma_{I}$. Let $u_{i}: Q \times \Sigma_{i} \rightarrow \mathbf{R}$ be $i$ 's utility function. A family of parameterized societies is a list of specified data, $\left(\left\{\Sigma_{i}\right\}_{i \in I}, Q,\left\{\Phi_{S}\right\}_{S \in I}, \Psi,\left\{u_{i}\right\}_{i \in I}\right)$. A social coalitional equilibrium of the parameterized family of societies is a pair of a parameter value and a strategy bundle $\left(q^{*}, \sigma_{I}^{*}\right) \in Q \times \Sigma$ such that (i) $\sigma_{I}^{*} \in \Phi_{I}\left(q^{*}\right), q^{*} \in \Psi\left(q^{*}, \sigma_{I}^{*}\right)$, and (ii) it is not true that there exist $S \in \mathcal{I}$ and $\sigma_{S} \in \Phi_{S}\left(q^{*}\right)$ for which $u_{i}\left(q^{*}, \sigma_{i}\right)>u_{i}\left(q^{*}, \sigma_{i}^{*}\right)$ for every $i \in S$.

THEOREM 6.5 Let $\left(\left\{\Sigma_{i}\right\}_{i \in I}, Q,\left\{\Phi_{S}\right\}_{S \in \mathcal{I}}, \Psi,\left\{u_{i}\right\}_{i \in I}\right)$ be a family of parameterized societies. There exists a social coalitional equilibrium if: 
(i) $\Sigma_{i}$ is a nonempty, convex, compact subset of a Euclidean space for every $i \in I$, and so is $Q$;

(ii) for every $S \in \mathcal{I}, \Phi_{S}$ is both upper semicontinuous and lower semicontinuous in $Q$, and is nonempty- and closed-valued;

(iii) for every $i \in I, u_{i}$ is continuous in $Q \times \Sigma_{i}$;

(iv) for every $\bar{q} \in Q$, the non-side-payment game $V_{\bar{q}}: \mathcal{I} \rightarrow \mathbf{R}^{I}$ defined by

$$
V_{\bar{q}}(S):=\left\{u \in \mathbf{R}^{I} \mid \exists \sigma_{S} \in \Phi_{S}(\bar{q}): \forall i \in S: u_{i} \leq u_{i}\left(\bar{q}, \sigma_{i}\right)\right\}
$$

is balanced;

(v) for every $\bar{q} \in Q$ and for every utility allocation $\bar{u}$ in the core of $V_{\bar{q}}$, the set $\left\{\sigma_{I} \in \Phi_{I}(\bar{q}) \mid \forall i \in I: \bar{u}_{i} \leq u_{i}\left(\bar{q}, \sigma_{i}\right)\right\}$ is convex; and

(vi) $\Psi$ is upper semicontinuous in $Q \times \Sigma$, and is nonempty-, closed- and convex-valued.

Theorem 4.2 will be proved by applying the technique of Ichiishi and Quinzii (1983) modified suitably for the present purpose: Roughly stated, a family of societies $\Gamma:=\left(\left\{\Sigma_{i}\right\}_{i \in I}, Q,\left\{\Phi_{S}\right\}_{S \in I}, \Psi,\left\{u_{i}\right\}_{i \in I}\right)$ parameterized by price vector $q \in Q$ is constructed from the profit-center game $\left(\left\{T_{i}, \pi_{i}\right\}_{i \in I}\right.$, $\left.\left\{Y_{i}, r_{i}(\cdot)\right\}_{i \in I}, p\right)$. The parameterized family $\Gamma$ will be shown to satisfy the assumptions of Theorem 6.5. From a social coalitional equilibrium of $\Gamma$, one can easily construct the required core plan of the original profit-center game. Recall, however, the cost-minimization problem $P_{I}(q, \eta)$ and the partition of the price domain into $\left\{Q_{+}, Q_{0}\right\}$, defined in Section 4. To be precise, in view of the fact that Problem $P_{I}(q, \eta)$ may not have an optimal solution if $q \in Q_{0}$, a sequence of parameterized families of societies $\left\{\Gamma^{\nu}\right\}_{\nu=1}^{\infty}$ is actually constructed, a social coalitional equilibrium $\left(q^{\nu}, \sigma_{I}^{\nu}\right)$ of $\Gamma^{\nu}$ is chosen for each $\nu$, and a limit point $\left(\bar{q}, \bar{\sigma}_{I}\right)$ of the sequence $\left\{q^{\nu}, \sigma_{I}^{\nu}\right\}_{\nu}$ is considered.

The definition of $\Gamma^{\nu}$ is now presented. The fictitious price vector $q$ for the type-profile-contingent nonmarket commodities, introduced at the outset of Section 4, will play the role of parameter in $\Gamma^{\nu}$. Define

$$
Q_{\nu}:=\left\{q \in Q \mid \forall t \in T: \forall a \in K_{n}: q(t, a) \geq \frac{1}{\nu}\right\} .
$$

Since $Q_{\nu} \subset Q_{+}$, Problem $P_{I}(q, \eta)$ has an optimal solution for all $(q, \eta) \in$ $Q_{\nu} \times \stackrel{\circ}{\mathbf{R}}_{+}$. The parameter space of $\Gamma^{\nu}$ is, therefore, the trimmed simplex $Q_{\nu}$. 
Define a lower bound and an upper bound for the maximal profit:

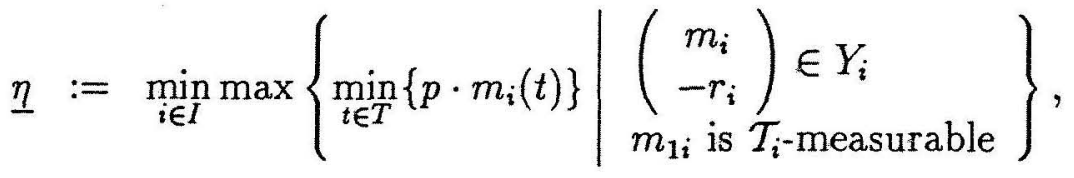

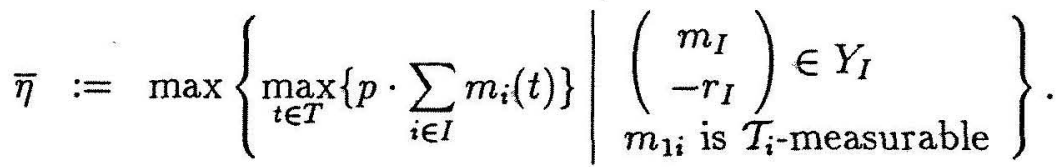

From Assumption 2.6 (iv) and the present conditions ((1) positive profitability from strict inputs and (2) strict positiveness of $\left.r_{i}\left(t_{i}\right)\right)$, it follows that $0<\underline{\eta} \leq \bar{\eta}<\infty$. The strategy spaces are defined as

$$
\Sigma_{i}:=[\underline{\eta}, \bar{\eta}] \times\{0\}\left(\subset \stackrel{\circ}{+}_{+} \times \mathbf{R}_{+}\right), i \in I .
$$

Division $i$ 's strategy $\left(\eta_{i}, \zeta_{i}\right) \in \Sigma_{i}$ is identified with $\eta_{i} \in[\underline{\eta}, \bar{\eta}]$ since $\zeta_{i} \equiv 0$, and is interpreted as the profit attributed to it. To use the language of the model of Theorem 6.4, $\left(\eta_{i}, \zeta_{i}\right)$ means $i$ 's consumption of $\eta_{i}$ units of the "final good", and no consumption of the "primary good". Define $\Sigma_{S}:=\prod_{i \in S} \Sigma_{i}$, and set $\Sigma:=\Sigma_{I}$.

The feasible-strategy correspondences $\Phi_{S}: Q \rightarrow \Sigma_{S}, S \in \mathcal{I}$, are defined as

$$
\Phi_{S}(\bar{q}):=\left\{\left(\eta_{S}, 0\right) \in \Sigma_{S} \mid c_{S}^{\text {wic }}\left(\bar{q}, \sum_{i \in S} \eta_{i}\right) \leq \sum_{i \in S} \sum_{t \in T} \bar{q}(t) \cdot r_{i}\left(t_{i}\right)\right\} .
$$

(To be precise, the restriction of $\Phi_{S}$ to $Q_{\nu}$ is used for $\Gamma^{\nu}$.)

The utility functions of $\Gamma^{\nu}$ are given as

$$
u_{i}\left(q, \eta_{i}, \zeta_{i}\right):=\eta_{i}, i \in I .
$$

LEMMA 6.6 There exists a continuous function

$$
\begin{aligned}
\left(x_{I}^{*}, y^{*}\right): Q_{+} \times \stackrel{\circ}{\mathbf{R}}_{+} & \rightarrow \mathbf{R}^{|T \times I|} \times \mathbf{R}^{k|T|} \\
(q, \eta) & \mapsto\left(x_{I}^{*}(q, \eta)(\cdot), y^{*}(q, \eta)(\cdot)\right)
\end{aligned}
$$

such that for each $(q, \eta),\left(x_{I}^{*}(q, \eta), y^{*}(q, \eta)\right): T \rightarrow \mathbf{R}^{|I|} \times \mathbf{R}^{k}$ is an optimal solution to Problem $P_{I}(q, \eta)$. 
Proof Step 1. Given $q \in Q_{+}$and any function $x: T \rightarrow \mathbf{R}$, the problem,

$$
\begin{aligned}
\text { Minimize } & \sum_{t \in T} q(t) \cdot(-n(t)), \\
\text { subject to } & -n \in S_{Y_{I}}(m), \text { and } \\
& \forall t: x(t) \leq p \cdot m(t),
\end{aligned}
$$

has a unique solution $y^{*}(q, x)$. Indeed, let $y:=(m, n)$ and $y^{\prime}:=\left(m^{\prime}, n^{\prime}\right)$ be solutions. Then,

$$
g(q, m)=g\left(q, m^{\prime}\right) .
$$

If $m \neq m^{\prime}$, set $m^{\prime \prime}:=\left(m+m^{\prime}\right) / 2$. By strict quasi-convexity of $g(q, \cdot)$,

$$
g\left(q, m^{\prime \prime}\right)<g(q, m)
$$

and clearly

$$
\forall t \in T: x(t) \leq p \cdot m^{\prime \prime}(t),
$$

which contradicts the optimality of $y$. Therefore $m=m^{\prime}$. By strict convexity of $S_{Y_{I}}(m), n=n^{\prime}$.

Step 2. Using the subvector $n^{*}(q, x)$ of $y^{*}(q, x)$ obtained in Step 1, Problem $P_{I}(q, \eta)$ is equivalent to:

$$
\begin{aligned}
\text { Minimize } & -\sum_{t \in T} q(t) \cdot n^{*}\left(q, \sum_{i \in I} x_{i}\right)(t), \\
\text { subject to } & \eta \leq \sum_{i \in I} \min _{t \in T} E\left(x_{i} \mid \mathcal{T}_{i}\right)(t) .
\end{aligned}
$$

Let $x_{I}$ and $x_{I}^{\prime}$ be two optimal solutions of this problem. Then, one may assume without loss of generality,

$$
\forall t: \sum_{i \in I} x_{i}(t)=\sum_{i \in I} x_{i}^{\prime}(t)=: x^{*}(q, \eta)(t) .
$$

To show this, notice first that

$$
m^{*}\left(q, \sum_{i \in I} x_{i}\right)=m^{*}\left(q, \sum_{i \in I} x_{i}^{\prime}\right)
$$

Indeed, if this equality is false, set

$$
\begin{aligned}
x_{I}^{\prime \prime} & :=\frac{x_{I}+x_{I}^{\prime}}{2} \\
m^{\prime \prime} & :=\frac{m^{*}\left(q, \sum_{i \in I} x_{i}\right)+m^{*}\left(q, \sum_{i \in I} x_{i}^{\prime}\right)}{2} .
\end{aligned}
$$


Then,

$$
\begin{gathered}
\forall t: x^{\prime \prime}(t) \leq p \cdot m^{\prime \prime}(t), \\
\eta \leq \sum_{i \in I} \min _{t \in T} E\left(x_{i}^{\prime \prime} \mid \mathcal{T}_{i}\right)(t), \\
g\left(q, m^{\prime \prime}\right)<g\left(q, m^{*}\left(q, \sum_{i \in I} x_{i}\right)\right),
\end{gathered}
$$

which contradicts the optimality of $\left(x_{I}, m^{*}\left(q, \sum_{i \in I} x_{i}\right)\right)$. One can then define

$$
\forall t: x^{*}(q, \eta)(t):=p \cdot m^{*}\left(q, \sum_{i \in I} x_{i}\right)(t)
$$

Step 3. By the maximum theorem, the function $(q, \eta) \mapsto x^{*}(q, \eta)(\cdot)$ is continuous on $Q_{+} \times \stackrel{\circ}{\mathbf{R}}_{+}$. The set of optimal solutions of the problem $P_{I}(q, \eta)$ is now given as

$$
\left\{\begin{array}{l|l}
x_{I}: T \rightarrow \mathbf{R}^{I} & \begin{array}{l}
\forall t \in T: \\
\sum_{i \in I} E\left(x_{i} \mid t_{i}\right) \geq \eta \\
\sum_{i \in I} x_{i}(t) \leq x^{*}(q, \eta)(t)
\end{array}
\end{array}\right\}
$$

By Walkup and Wets (1969), this polyhedron depends continuous piecewise linearly on the right-hand-side parameter of the constraint, $\left(\eta, x^{*}(q, \eta)(\cdot)\right)$; that is, there are finitely many continuous piecewise linear functions $h^{j}, j \in$ $J$, such that for each $\left(\eta, x^{*}(q, \eta)(\cdot)\right)$,

$\left\{\right.$ the extreme points of the polyhedron given $\left.\left(\eta, x^{*}(q, \eta)(\cdot)\right)\right\}$

$$
\subset\left\{h^{j}\left(\eta, x^{*}(q, \eta)(\cdot)\right) \mid j \in J\right\} \text {. }
$$

In particular, each extreme point depends continuously on $(q, \eta)$. This establishes the required choice of $x_{I}^{*}: Q_{+} \times \stackrel{\circ}{\mathbf{R}}_{+} \rightarrow \mathbf{R}^{|T \times I|}$.

Step 4. Define

$$
y^{*}(q, \eta)(\cdot):=y^{*}\left(q, x^{*}(q, \eta)(\cdot)\right)(\cdot)
$$

where $y^{*}$ of the right-hand side is given in Step 1 and $x^{*}$ is given in Step 2 .

The correspondence $\Psi^{\nu}: Q_{\nu} \times \Sigma \rightarrow Q_{\nu}$ of the parameterized society $\Gamma^{\nu}$ is defined by: $q^{\circ} \in \Psi^{\nu}\left(\bar{q},\left(\bar{\eta}_{I}, 0\right)\right)$ iff $q^{\circ}$ maximizes the value of the "total 
excess demand for the 'nonmarket commodities' ". That is, $q^{\circ}$ solves the mathematical programming problem,

$$
\begin{array}{ll}
\text { Maximize } & \sum_{t \in T} q(t) \cdot\left(-n^{*}\left(\bar{q}, \sum_{j \in I} \bar{\eta}_{j}\right)(t)-\sum_{i \in I} r_{i}\left(t_{i}\right)\right), \\
\text { subject to } & q \in Q_{\nu},
\end{array}
$$

where $n^{*}\left(\bar{q}, \sum_{j \in I} \bar{\eta}_{j}\right)(\cdot): T \rightarrow \mathbf{R}^{k_{n}}$ is given in Lemma 6.6. Solution $q^{\circ}(\epsilon$ $\left.\Psi\left(\bar{q},\left(\bar{\eta}_{I}, 0\right)\right)\right)$ assigns a large weight $\left(\right.$ i.e., $\left.q^{\circ}(\bar{t}, \bar{a})>1 / \nu\right)$ only if the excess demand for $(\bar{t}, \bar{a})$ is the greatest for all $(t, a) \in T \times K_{n}$.

Proof of Theorem 4.2 Step 1. For each $\nu$ large enough so that $Q_{\nu} \neq \emptyset$, the family of parameterized societies

$$
\Gamma^{\nu}:=\left(\left\{\Sigma_{i}\right\}_{i \in I}, Q_{\nu},\left\{\Phi_{S}\right\}_{S \in I}, \Psi^{\nu},\left\{u_{i}\right\}_{i \in I}\right)
$$

that is constructed from the profit-center game

$$
\left(\left\{T_{i}, \pi_{i}\right\}_{i \in I},\left\{Y_{i}, r_{i}(\cdot)\right\}_{i \in I}, p\right)
$$

is well-defined and satisfies conditions (i) and (iii) of Theorem 6.5.

One may assume without loss of generality that $c_{S}^{w i c}=c_{I}^{w i c}$ for all $S$. Indeed, by Assumption 2.6 (ii), $c_{S}^{w i c} \geq c_{I}^{w i c}$ for all $S$. A core plan of the (hypothetical) profit-center game in which every coalition $S$ has access to the cost function $c_{I}^{\text {wic }}$ is also a core plan of the (original) profit-center game, since in the latter game the "blocking power" of $S$ is weaker, so more plans are coalitionally stable than in the former game.

The family $\Gamma^{\nu}$ satisfies condition (ii) of Theorem 6.5. Indeed, In view of Assumption 4.1 (iii) and strict positiveness of $r_{i}\left(t_{i}\right)$, the standard technique in the neoclassical consumer theory to establish upper and lower semicontinuity of the budget-set correspondence is applicable.

The family $\Gamma^{\nu}$ satisfies condition (iv) of Theorem 6.5. Indeed, by Assumption 4.1 (i), for each given $q \in Q$,

$$
0<\eta<\eta^{\prime} \Longrightarrow \frac{c_{I}^{w i c}(q, \eta)}{\eta} \geq \frac{c_{I}^{w i c}\left(q, \eta^{\prime}\right)}{\eta^{\prime}}
$$

Therefore, for each $q \in Q$, the non-side-payment game $V_{q}^{\text {wic }}: \mathcal{I} \rightarrow \mathbf{R}^{I}$ defined by

$$
V_{q}^{\text {wic }}(S):=\left\{u \in \mathbf{R}^{I} \mid \exists \sigma_{S} \in \Phi_{S}(q): \forall i \in S: u_{i} \leq u_{i}\left(q, \sigma_{i}\right)\right\}
$$


is balanced by Theorem 6.4 .

The family $\Gamma^{\nu}$ satisfies condition (v) of Theorem 6.5. Indeed, choose any $\bar{q} \in Q_{\nu}$ and any utility allocation $\bar{u} \in \mathbf{R}^{I}$. Let $\sigma_{I}^{0}, \sigma_{I}^{1} \in \Phi_{I}(\bar{q})$ be such that

$$
\forall i \in I: \bar{u}_{i} \leq \min \left\{u_{i}\left(\bar{q}, \sigma_{i}^{0}\right), u_{i}\left(\bar{q}, \sigma_{i}^{1}\right)\right\},
$$

and for each $\alpha \in[0,1]$ define $\sigma_{I}^{\alpha} \in \Sigma$ by

$$
\sigma_{I}^{\alpha}:=\alpha \sigma_{I}^{1}+(1-\alpha) \sigma_{I}^{0} .
$$

Clearly, $\ddot{\bar{u}}_{i} \leq u_{i}\left(\bar{q}, \sigma_{i}^{\alpha}\right)$ for all $i \in I$. It suffices to show that $\sigma_{I}^{\alpha} \in \Phi_{I}(\bar{q})$. Let $\left(x_{I}^{0}, y^{0}\right) \in C_{I}\left(\sum_{i \in I} \eta_{i}^{0}\right)$ be an optimal solution to Problem $P_{I}\left(\bar{q}, \sum_{i \in I} \eta_{i}^{0}\right)$.

Then,

$$
g\left(\bar{q}, m^{0}\right)=c_{I}^{w i c}\left(\bar{q}, \sum_{i \in I} \eta_{i}^{0}\right) \leq \sum_{i \in I} \sum_{t \in T} \bar{q}(t) \cdot r_{i}\left(t_{i}\right) .
$$

Similarly, for an optimal solution $\left(x_{I}^{1}, y^{1}\right)$ to Problem $P_{I}\left(\bar{q}, \sum_{i \in I} \eta_{i}^{1}\right)$,

$$
g\left(\bar{q}, m^{1}\right) \leq \sum_{i \in I} \sum_{t \in T} \bar{q}(t) \cdot r_{i}\left(t_{i}\right) .
$$

Define

$$
\left(x_{I}^{\alpha}, m^{\alpha}\right):=\alpha\left(x_{I}^{1}, m^{1}\right)+(1-\alpha)\left(x_{I}^{0}, m^{0}\right) .
$$

By Assumption 4.1 (iv), for any $\alpha \in[0,1]$,

$$
g\left(\bar{q}, m^{\alpha}\right) \leq \sum_{i \in I} \sum_{t \in T} \bar{q}(t) \cdot r_{i}\left(t_{i}\right)
$$

On the other hand, for any $n_{i}$ for which $n_{1 i}$ is $\mathcal{T}_{i}$-measurable and $\left(m^{\alpha}, \sum_{i \in I} n_{i}\right) \in$ $\sum_{i \in I} Y_{i}$, it follows that $\left(x_{I}^{\alpha}, m^{\alpha}, \sum_{i \in I} n_{i}\right) \in C_{I}\left(\sum_{i \in I} \eta_{i}^{\alpha}\right)$, so that

$$
c_{I}^{w i c}\left(\bar{q}, \sum_{i \in I} \eta_{i}^{\alpha}\right) \leq-\sum_{i \in I} \sum_{t \in T} \bar{q}(t) \cdot n_{i}(t)
$$

and consequently,

$$
c_{I}^{w i c}\left(\bar{q}, \sum_{i \in I} \eta_{i}^{\alpha}\right) \leq g\left(\bar{q}, m^{\alpha}\right)
$$

Therefore,

$$
c_{I}^{w i c}\left(\bar{q}, \sum_{i \in I} \eta_{i}^{\alpha}\right) \leq \sum_{i \in I} \sum_{t \in T} \bar{q}(t) \cdot r_{i}\left(t_{i}\right),
$$

that is, $\sigma_{I}^{\alpha} \in \Phi(\bar{q})$. 
The family $\Gamma^{\nu}$ satisfies condition (vi) of Theorem 6.5 by the maximum theorem.

Thus, all the assumptions of Theorem 6.5 are satisfied. Let $\left(q^{\nu}, \sigma_{I}^{\nu}\right) \in$ $Q_{\nu} \times \Sigma$ be a social coalitional equilibrium of $\Gamma^{\nu}$. By definition of $\Psi^{\nu}, q^{\nu} \in Q_{+}$. Let

$$
\left(x_{I}^{\nu}, y^{\nu}\right):=\left(x_{I}^{*}\left(q^{\nu}, \sum_{i \in I} \eta_{i}^{\nu}\right), y^{*}\left(q^{\nu}, \sum_{i \in I} \eta_{i}^{\nu}\right)\right)
$$

it is an optimal solution to Problem $P_{I}\left(q^{\nu}, \sum_{i \in I} \eta_{i}^{\nu}\right)$. Let $y_{I}^{\nu} \in Y_{I}$ give rise to $y^{\nu}$ (in particular, $y^{\nu}=\sum_{i \in I} y_{i}^{\nu}$ ). One may choose $y_{I}^{\nu}$ so that each $y_{1 i}^{\nu}$ is $\mathcal{T}_{i}$-measurable.

Step 2. The sequence $\left\{n^{\nu}\right\}_{\nu}\left(\subset-\mathbf{R}_{+}^{k_{2}|T|}\right)$ obtained in Step 1 is bounded. To show this, suppose the contrary. Then, there exists a subsequence, still denoted by $\left\{n^{\nu}\right\}_{\nu}$, such that

$$
n^{\nu}\left(t^{\nu}, a^{\nu}\right) \rightarrow-\infty, \quad \text { as } \quad \nu \rightarrow \infty,
$$

for some choice of coordinate $\left(t^{\nu}, a^{\nu}\right)$ for each $\nu$. For each $\nu$, define $L^{\nu}$ (C $T \times K_{n}$ ) as the set of (type-profile, commodity)-pairs whose excess demand is maximal. That is, $(\bar{t}, \bar{a}) \in L^{\nu}$ iff

$$
-n^{\nu}(\bar{t}, \bar{a})-\sum_{i \in I} r_{i}\left(\bar{t}_{i}, \bar{a}\right)=\max _{(t, a)}\left\{-n^{\nu}(t, a)-\sum_{i \in I} r_{i}\left(t_{i}, a\right)\right\} .
$$

By passing through a subsequence if necessary, one may assume without loss of generality that

$$
L^{\nu}=L^{\nu+1}=\cdots=: L^{\circ} .
$$

By the present hypothesis (7),

$$
\forall(t, a) \in L^{\circ}: n^{\nu}(t, a) \rightarrow-\infty, \text { as } \nu \rightarrow \infty .
$$

This holds true, only if $\left(q^{\nu}, \sigma_{I}^{\nu}\right)$ as a point in the domain $Q_{\nu} \times \Sigma$ of the correspondence $\Psi^{\nu}$ satisfies

$$
\forall(t, a) \in L^{\circ}: q^{\nu}(t, a) \rightarrow 0, \text { as } \nu \rightarrow \infty
$$

(otherwise, the cost $-\sum_{t \in T} q^{\nu}(t) \cdot n^{\nu}(t)$ would be arbitrarily large, which contradicts the definition of $n^{\nu}$ as a cost-minimizer). Therefore,

$$
\sum_{(t, a) \in L^{\circ}} q^{\nu}(t, a) \rightarrow 0, \quad \text { as } \quad \nu \rightarrow \infty
$$


On the other hand, $q^{\nu}$ as a point in the response set $\Psi^{\nu}\left(q^{\nu}, \sigma_{I}^{\nu}\right)$ assigns a greater weight than $1 / \nu$ only to members of $L^{\circ}$. So

$$
\begin{aligned}
\sum_{(t, a) \in L^{\circ}} q^{\nu}(t, a) & \geq 1-\frac{k_{n}|T|}{\nu} \\
& \rightarrow 1, \text { as } \nu \rightarrow \infty,
\end{aligned}
$$

which contradicts (8).

Step 3. Since $\left\{n^{\nu}\right\}_{\nu}$ is bounded, and since

$$
\begin{aligned}
n^{\nu} & =\sum_{i \in I} n_{i}^{\nu}, \\
n_{i}^{\nu} & \leq \quad \text { for every } i \in I,
\end{aligned}
$$

the sequence $\left\{n_{i}^{\nu}\right\}_{\nu}$ is also bounded for each $i$. By Assumption 2.6 (iv), $\left\{m_{i}^{\nu}\right\}_{\nu}$ is bounded from above, and hence so is $\left\{m^{\nu}\right\}_{\nu}$. Moreover,

$$
\forall t \in T: \eta^{\nu} \leq p \cdot E\left(m^{\nu} \mid \mathcal{T}_{i}\right)(t),
$$

so $\left\{m^{\nu}\right\}_{\nu}$ is bounded from below as well. Therefore, $\left\{m_{i}^{\nu}\right\}_{\nu}$ is also bounded. Since $\left(x_{I}^{\nu}, y^{\nu}\right) \in C_{I}\left(\eta^{\nu}\right),\left\{x_{I}^{\nu}\right\}_{\nu}$ is bounded. Thus, one may assume without loss of generality,

$$
\begin{aligned}
q^{\nu} & \rightarrow \bar{q} \in Q, \\
\sigma_{I}^{\nu} & \rightarrow \bar{\sigma}_{I} \in \Sigma . \\
x_{I}^{\nu} & \rightarrow \bar{x}_{I} \in \mathbf{R}^{|T \times I|}, \\
y_{I}^{\nu} & \rightarrow \bar{y}_{I} \in Y_{I}, \text { as } \nu \rightarrow \infty .
\end{aligned}
$$

Step 4. The plan $\left(\bar{x}_{I}, \bar{y}_{I}\right)$ is a member of $F_{I}$. To show this, one only needs to check

$$
\forall t \in T: \quad-\sum_{i \in I} \bar{n}_{i}(t) \leq \sum_{i \in I} r_{i}\left(t_{i}\right) .
$$

For this purpose, choose any $q$ in the relative interior of $Q$. Then, $q \in Q_{\nu}$ for all $\nu$ sufficiently large, so that

$$
\sum_{t \in T} q(t) \cdot\left(-\sum_{i \in I} n_{i}^{\nu}(t)-\sum_{i \in I} r_{i}\left(t_{i}\right)\right)
$$




$$
\begin{aligned}
& \leq \sum_{t \in T} q^{\nu}(t) \cdot\left(-\sum_{i \in I} n_{i}^{\nu}(t)-\sum_{i \in I} r_{i}\left(t_{i}\right)\right) \\
& =c_{I}^{\text {wic }}\left(q^{\nu}, \sum_{i \in I} \eta_{i}^{\nu}\right)-\sum_{i \in I} \sum_{t \in T} q^{\nu}(t) \cdot r_{i}\left(t_{i}\right) \\
& \leq 0 .
\end{aligned}
$$

Letting $\nu \rightarrow \infty$,

$$
\sum_{t \in T} q(t) \cdot\left(-\sum_{i \in I} \bar{n}_{i}(t)-\sum_{i \in I} r_{i}\left(t_{i}\right)\right) \leq 0 .
$$

This is true for all $q$ in the relative interior of $Q$, hence it is also true for all $q \in Q$. In particular, it is true for $q=e^{\tilde{t}, \tilde{a}} \in Q$ defined by

$$
e^{\tilde{t}, \tilde{a}}(t, a):= \begin{cases}1, & \text { if }(t, a)=(\tilde{t}, \tilde{a}), \\ 0, & \text { otherwise. }\end{cases}
$$

This means that

$$
-\sum_{i \in I} \bar{n}_{i}(\tilde{t}, \tilde{a})-\sum_{i \in I} r_{i}\left(\tilde{t}_{i}, \tilde{a}\right) \leq 0 .
$$

Since $(\tilde{t}, \tilde{a})$ was chosen arbitrarily, $(9)$ is now established.

Since $\eta_{I}^{\nu}\left(:=\left(u_{i}\left(q^{\nu}, \sigma_{i}^{\nu}\right)\right)_{i \in I}\right)$ is in the core of game $V_{q^{\nu}}^{\text {wic }}$ for every $\nu$, and since $\Phi_{S}$ 's are upper and lower semicontinuous, it follows that $\bar{\eta}_{I}$ is in the core of game $V_{\bar{q}}^{\text {wic }}$ :

$$
\bar{\eta}_{I} \in V_{\bar{q}}^{w i c}(I)
$$

and

$$
\neg \exists S \in \mathcal{I}: \exists \eta_{I} \in V_{\bar{q}}^{\text {wic }}(S): \forall i \in S: \eta_{i}>\bar{\eta}_{i} .
$$

By $(10),\left(\bar{x}_{I}, \bar{y}_{I}\right) \in C_{I}\left(\sum_{i \in I} \bar{\eta}_{i}\right)$. Define a profit-imputation plan $\bar{x}_{I}^{\prime}$ by:

$$
\bar{x}_{i}^{\prime}\left(t_{i}, t_{I \backslash\{i\}}\right):=\bar{\eta}_{i}-E\left(\bar{x}_{i} \mid \mathcal{T}_{i}\right)+\bar{x}_{i}\left(t_{i}, t_{I \backslash\{i\}}\right) .
$$

Then,

$$
\begin{aligned}
E\left(\bar{x}_{i}^{\prime} \mid \mathcal{T}_{i}\right)(t) & =\bar{\eta}_{i}-E\left(\bar{x}_{i} \mid \mathcal{T}_{i}\right)(t)+E\left(\bar{x}_{i} \mid \mathcal{T}_{i}\right)(t) \\
& =\bar{\eta}_{i}
\end{aligned}
$$


which is independent of $t$. Moreover, for all $t \in T$,

$$
\begin{aligned}
\sum_{i \in I} \bar{x}_{i}^{\prime}(t) & =\sum_{i \in I} \bar{\eta}_{i}-\sum_{i \in I} E\left(\bar{x}_{i} \mid \mathcal{T}_{i}\right)(t)+\sum_{i \in I} \bar{x}_{i}(t) \\
& \leq \sum_{i \in I} \bar{x}_{i}(t) \\
& \leq \sum_{i \in I} p \cdot \bar{m}_{i}(t) .
\end{aligned}
$$

Therefore, $\left(\bar{x}_{I}^{\prime}, \bar{y}_{I}\right) \in G_{I}^{w i c}$. Define $\overline{\bar{x}}_{i}:=E\left(\bar{x}_{i}^{\prime} \mid \mathcal{T}_{i}\right)$. Then, $\overline{\bar{x}}_{i}(t) \equiv \bar{\eta}_{i}$. By (11) no coalition $S$ can improve upon $\left(\overline{\bar{x}}_{I}, \bar{y}_{I}\right)$ using strategies in $G_{S}^{\text {wic. }}$.

Using Lemma 6.3 and Postulate 2.4, one can construct the required fullinformation revealing Bayesian incentive-compatible core plan from $\left(\bar{x}_{I}^{\prime}, \bar{y}_{I}\right)$, as in the last three paragraphs of the proof of Theorem 3.1.

Proof of Theorem 5.2 is a straightforward consequence of the following Lemma 6.7 (applied to game $V^{\text {wic }}$ ) and the argument in the last three paragraphs of the proof of Theorem 3.1, so the details are left to the reader. Let $\partial V(I)$ be the Pareto frontier of set $V(I)$. Assumption (iii) of Lemma 6.7 means that $\partial V(I)$ is strictly negatively sloped.

LEMMA 6.7 Let $V: \mathcal{I} \rightarrow \mathbf{R}^{I}$ be a non-side-payment game. Assume:

(i) $V$ is superadditive, i.e., for any disjoint coalitions $S_{1}$ and $S_{2}$ it follows that $V\left(S_{1}\right) \cap V\left(S_{2}\right) \subset V\left(S_{1} \cup S_{2}\right)$.

(ii) There exist a partition $\left\{I_{s}, I_{c}\right\}$ of $I$, and $\underline{u} \in \mathbf{R}^{I}$ such that if $S \not \supset I_{s}$, then $V(S)=\left[\left\{\underline{u}_{S}\right\}-\mathbf{R}_{+}^{S}\right] \times \mathbf{R}^{I \backslash S}$.

(iii) For any $\varepsilon>0$ and any $u \in \partial V(I)$ for which $u>\underline{u}$, there exist $u^{\prime} \in V(I)$ and $i \in\left\{j \in I \mid u_{j}>\underline{u}_{j}\right\}$, such that $u_{i}^{\prime}=u_{i}-\varepsilon$ and $u_{j}^{\prime}>u_{j}$ for all $j \in I \backslash\{i\}$. Choose any $u^{*} \in \partial V(I)$ such that

$$
\begin{array}{ll}
u_{i}^{*} \geq \underline{u}_{i} & \text { for all } i \in I_{s}, \quad \text { and } \\
u_{i}^{*}=\underline{u}_{i} & \text { for all } i \in I_{c} .
\end{array}
$$

Then $u^{*} \in C(V)$.

Proof Suppose there exists $S \in \mathcal{I}$ such that $u^{*} \in \dot{V}^{\circ}(S)$. Then

$$
I_{c} \subset S \neq I \text {. }
$$


Let $u^{\prime} \in V(S)$ be such that

$$
\begin{array}{ll}
u_{i}^{\prime}>u_{i}^{*} & \text { for all } i \in S, \\
u_{i}^{\prime}=\underline{u}_{i} & \text { for all } i \in I \backslash S\left(\subset I_{c}\right) .
\end{array}
$$

By the superadditivity, $u^{\prime} \in V(I)$. By the strict negative-slopedness of $\partial V(I)$, there exists $u^{\prime \prime} \in V(I)$ such that $u^{\prime \prime} \gg u^{*}$, which contradicts the choice of $u^{*}$ as a point of $\partial V(I)$.

\section{Acknowledgements}

Discussions and comments of Birgit Grodal, Joe Harrington, Jim Peck, Bob Rosenthal and Jingang Zhao on earlier drafts of the paper are gratefully acknowledged. The material here is based upon work supported in part by the NSF under Award No. INT-9121247.

\section{References}

Chandler, Jr., A. D., 1962, Strategy and structure (MIT Press, Cambridge, MA).

d'Aspremont, C. and L.-A. Gérard-Varet, 1979, Incentives and incomplete information, Journal of Public Economics 11, 25-45.

Ichiishi, T., A. Idzik and J. Zhao, 1994, Cooperative processing of information via choice at an information Set, International Journal of Game Theory $23,145-165$.

Ichiishi, T. and M. Quinzii, 1983, Decentralization for the core of a production economy with increasing returns, International Economic Review $24,397-412$.

Ichiishi, T. and M. R. Sertel, forthcoming, Cooperative interim contract and re-contract: Chandler's M-form firm, Economic Theory.

Klein, B., R. Crawford and A. A. Alchian, 1978, Vertical integration, appropriable rents, and the competitive contracting process, Journal of Law and Economics 21, 297-326.

Radner, R., 1979, Rational expectations equilibrium generic existence and the information revealed by prices, Econometrica 47, 655-678. 
Radner, R., 1992, Transfer payments and the core of a profit-center game, in: P. Dasgupta, et al., Economic analysis of markets and games (Essays in honor of Frank Hahn) (MIT Press, Cambridge, MA) 316-339.

Scarf, H., 1973, The computation of economic equilibria (Yale University Press, New Haven, CT).

Scarf, H., 1986, Notes on the core of a productive economy, in: W. Hildenbrand, et al., Contributions to mathematical economics (in Honor of Gerard Debreu) (North-Holland, Amsterdam/New York) 401-429.

Walkup, D. W. and R. J.-B. Wets, 1969, Lifting projections of convex polyhedra, Pacific Journal of Mathematics 28, 465-475.

Williamson, O. E., 1975 Markets and hierarchies: Analysis and antitrust implications (Free Press, New York, NY).

Williamson, O. E., 1991, Comparative economic organization: The analysis of discrete structural alternatives, Administrative Science Quarterly 36, 269-296. 